\title{
Holographic Aspects of a Relativistic Nonconformal Theory
}

\author{
Chanyong Park \\ Center for Quantum Spacetime (CQUeST), Sogang University, Seoul 121-742, Republic of Korea \\ Correspondence should be addressed to Chanyong Park; cyong21@gmail.com
}

Received 16 May 2013; Revised 24 July 2013; Accepted 30 July 2013

Academic Editor: Suvankar Dutta

Copyright (C) 2013 Chanyong Park. This is an open access article distributed under the Creative Commons Attribution License, which permits unrestricted use, distribution, and reproduction in any medium, provided the original work is properly cited.

\begin{abstract}
We study a general $D$-dimensional Schwarzschild-type black brane solution of the Einstein-dilaton theory and derive, by using the holographic renormalization, its thermodynamics consistent with the geometric results. Using the membrane paradigm, we calculate the several hydrodynamic transport coefficients and compare them with the results obtained by the Kubo formula, which shows the self-consistency of the gauge/gravity duality in the relativistic nonconformal theory. In order to understand more about the relativistic non-conformal theory, we further investigate the binding energy, drag force, and holographic entanglement entropy of the relativistic non-conformal theory.
\end{abstract}

\section{Introduction}

For the last decade, the AdS/CFT correspondence [1-3] has been one of the interesting subjects for understanding the strongly interacting quantum field theory (QFT). Applying the AdS/CFT correspondence to QCD or the condensed matter system provided many interesting physical results like the phase structures [4-7], the universal ratio between the shear viscosity and entropy [8-14], holographic superconductor [15-29] and strange metallic behavior [30-32], and so forth. Recently, after assuming the gauge/gravity duality it was shown that the dual field theory of the Einstein-dilaton gravity can be described by a relativistic nonconformal theory [33]. In this model, due to the running dilaton, the DC conductivity obtained by the Kubo formula $[8,9,33]$ shows the unexpected behavior, which may describe electrolyte or some chemical compounds. The real physics is not usually conformal except some critical phenomena like the phase transition and the RG fixed points. Therefore, it is required to generalize the AdS/CFT correspondence to the nonconformal case. Here, we simply call such a generalized correspondence the gauge/gravity duality. Actually, it is not hopeful to prove the gauge/gravity duality because even in the AdS space there is no direct proof of the AdS/CFT correspondence. Instead, we will try to find some pieces of evidences for the gauge/gravity duality of a nonconformal theory.

In the Einstein-dilaton theory with a Liouville potential, there exists a Schwarzschild-type black brane solution which we call an Einstein-dilaton black brane (EdBB). Since its asymptotic geometry is not the AdS space and the induced metric on the boundary is given by the Minkowski metric, the gauge/gravity duality says that the dual theory should be a relativistic nonconformal theory. In order to understand more physical properties, one can apply the holographic renormalization to the Einstein-dilaton theory. In this paper after finding an appropriate counterterm we show that the resulting on-shell action and the boundary stress tensor are finite. Furthermore, we check the self-consistency of the gauge/gravity duality by showing that the thermodynamic quantities derived from the boundary stress tensor coincide with the results of the EdBB geometry.

Another interesting issue related to the self-consistency is the hydrodynamics. In the linear response theory of the QFT, the macroscopic properties can be determined by the transport coefficients. Moreover, they can be represented by the background thermal quantities. For example, consider a thermal system with an energy density $\epsilon$ and pressure $P$. Then, the momentum diffusion constant of this system is given by (see [15-19] and references therein)

$$
\mathscr{D}_{s}=\frac{\eta_{s}}{\epsilon+P}
$$

where $\eta_{s}$ is the shear viscosity. Note that in this calculation the microscopic details of the system are not important. If the EdBB is really dual to a relativistic nonconformal theory, the 
dual system should also satisfy this relation. Using the universality of the ratio between the shear viscosity and the entropy density [11, 15-19]

$$
\frac{\eta_{s}}{s}=\frac{1}{4 \pi}
$$

as well as the thermodynamic results of the Einstein-dilaton theory, the momentum diffusion constant becomes

$$
\mathscr{D}_{s}(\infty)=\frac{1}{4 \pi T_{H}}
$$

An alternative way to obtain the momentum diffusion constant is to investigate the holographic hydrodynamics of the metric fluctuations. For the self-consistency those two results should be the same. We show by applying the membrane paradigm [11] that the momentum diffusion constant obtained by the holographic method really satisfies the above relation.

In order to understand more physical features of the relativistic nonconformal theory, we further investigate the holographic binding energies probed by an F1- and D1-string. From the boundary theory point of view, an F1- or D1-string represents the bound state of a pair of particles (fundamental excitations) or monopoles (solitons), respectively. In the AdS space, there is no physical difference between F1- and D1strings due to the conformality or the trivial dilaton profile. However, in the relativistic nonconformal theory a monopole is distinguished from a particle because of the non-trivial coupling constant. The holographic results show that the binding energies of particles and monopoles are stronger in the nonconformal theory than in the conformal one. We also investigate the drag forces of a particle and monopole in the nonconformal medium. For a relativistic particle and monopole, the momentum exponentially decreases as time evolves. The dissipation rate of the momentum is proportional to temperature with a positive power depending on the nonconformality. In the nonrelativistic case, the momentum decays with an inverse power law for a particle and with a power law for a monopole, in which the dissipation power is again determined by the nonconformality. Finally, we investigate the holographic entanglement entropy of the relativistic nonconformal theory. Recently, there was an interesting conjecture that in a small subsystem the entanglement temperature (or "effective temperature") has a universal feature proportional to the inverse of the size [34]. Such a universality of the entanglement temperature also appears in the holographic relativistic nonconformal theory.

The rest of the paper is organized as follows. In Section 2, we explain our conventions and summarize the black brane thermodynamics. For checking the gauge/gravity duality of the relativistic nonconformal theory, in Section 3, we rederive the same thermodynamics from the boundary energymomentum tensor constructed by the holographic renormalization. Applying the membrane paradigm in Section 4, we also show that the momentum diffusion constant obtained in the EdBB gives rise to the consistent result with the EdBB thermodynamics and satisfies the QFT relation (1). Based on these self-consistencies of the gauge/gravity duality in the relativistic nonconformal theory, we further investigate the binding energies of particles and monopoles in Section 5 and the drag forces in Section 6. In Section 7, by calculating the holographic entanglement entropy, we show that the universal feature of the entanglement temperature conjectured in [34] is still valid even in the relativistic nonconformal theory. Finally, we finish our work with some concluding remarks in Section 8.

\section{D-Dimensional Einstein-Dilaton Black Brane}

Let us consider a $D$-dimensional Einstein-dilaton theory with a Liouville potential in a Lorentzian signature

$$
S_{E d}=\frac{1}{16 \pi G} \int_{\mathscr{M}} d^{D} x \sqrt{-g}\left[\mathscr{R}-2(\partial \phi)^{2}-2 \Lambda e^{\eta \phi}\right]
$$

where $\Lambda$ is a negative constant. We simply call $\Lambda$ a cosmological constant because it really becomes a cosmological constant for $\eta=0$. In the above, $\eta$ is an arbitrary constant representing the nonconformality of the dual theory. Taking a logarithmic profile for the dilaton field

$$
\phi(r)=\phi_{0}-k_{0} \log r,
$$

where $\phi_{0}$ and $k_{0}$ are two integration constants, an effective cosmological constant $\Lambda_{\text {eff }} \equiv \Lambda e^{\eta \phi_{0}}$ without loss of generality can be set to be

$$
\Lambda_{\mathrm{eff}}=-\frac{4(D-2)\left[8(D-1)-(D-2) \eta^{2}\right]}{\left[8+(D-2) \eta^{2}\right]^{2}} .
$$

Note that since $\Lambda$ is negative $\Lambda_{\text {eff }}$ should also be negative. This fact implies that $\eta$ is below the Gubser bound, $\eta^{2}<8(D-$ $1) /(D-2))[35,36]$. Consequently, $\phi(r)$ is refined to

$$
\phi(r)=-k_{0} \log r .
$$

In terms of the refined dilaton field and the effective cosmological constant, the Einstein equation and the equation of motion for a dilaton become

$$
\begin{gathered}
\mathscr{R}_{\mu \nu}-\frac{1}{2} \mathscr{R}_{\mu \nu}+g_{\mu \nu} \Lambda_{\mathrm{eff}} e^{\eta \phi} \\
=2 \partial_{\mu} \phi \partial_{\nu} \phi-g_{\mu \nu}(\partial \phi)^{2}, \\
\frac{1}{\sqrt{-g}} \partial_{\mu}\left(\sqrt{-g} g^{\mu \nu} \partial_{\nu} \phi\right)=\frac{1}{2} \Lambda_{\mathrm{eff}} \eta e^{\eta \phi} .
\end{gathered}
$$

Together with the logarithmic dilaton profile, the EdBB metric satisfying the above two equations is given by

$$
d s^{2}=-r^{2 a_{1}} f(r) d t^{2}+\frac{d r^{2}}{r^{2 a_{1}} f(r)}+r^{2 a_{1}} \delta_{i j} d x^{i} d x^{j},
$$

with a black brane factor

$$
f(r)=1-\frac{r_{h}^{c}}{r^{c}}
$$


where $i$ and $j$ represent the spatial directions of the boundary space, and the other parameters are

$$
\begin{gathered}
k_{0}=\frac{2(D-2) \eta}{8+(D-2) \eta^{2}}, \\
a_{1}=\frac{8}{8+(D-2) \eta^{2}}, \\
c=\frac{8(D-1)-(D-2) \eta^{2}}{8+(D-2) \eta^{2}} .
\end{gathered}
$$

The asymptote of the EdBB metric reduces to

$$
d s^{2}=\frac{1}{r^{2 a_{1}}} d r^{2}+r^{2 a_{1}}\left(-d t^{2}+\delta_{i j} d x^{i} d x^{j}\right),
$$

where the Poincare symmetry $\operatorname{ISO}(1, D-2)$ of the boundary hypersurface at a fixed $r$ is manifest. This fact implies that the dual field theory should be described by a relativistic QFT. For $\eta=0$, the background geometry reduces to the asymptotic AdS space with an effective cosmological constant $\Lambda_{\text {eff }}$ :

$$
\Lambda_{\mathrm{eff}}=-\frac{(D-1)(D-2)}{2}
$$

which is exactly that of a general $D$-dimensional AdS space with a unit AdS radius $R=1$. For $\eta \neq 0$, the asymptotic geometry is not the AdS space anymore and instead reduces to the hyperscaling violation form [37-48]. Therefore, one can easily see that the dual field theory is not conformal.

Before concluding this section, let us summarize thermodynamics of the EdBB obtained from metric (10). The Hawking temperature and the Bekenstein-Hawking entropy are

$$
\begin{gathered}
T_{H}=\frac{1}{4 \pi} \frac{8(D-1)+(D-2) \eta^{2}}{8+(D-2) \eta^{2}} r_{h}^{\left(8-(D-2) \eta^{2}\right) /\left(8+(D-2) \eta^{2}\right)}, \\
S_{B H}=\frac{V_{D-2}}{4 G} r_{h}^{8(D-2) /\left(8+(D-2) \eta^{2}\right)},
\end{gathered}
$$

where $V_{D-2}$ means the spatial volume of the boundary space. Other thermodynamic quantities, the internal energy $E$ and the free energy $F$, are given by

$$
\begin{aligned}
E= & \frac{V_{D-2}}{8 \pi G} \frac{4(D-2)}{8+(D-2) \eta^{2}} \\
& \times r_{h}^{\left(8(D-1)-(D-2) \eta^{2}\right) /\left(8+(D-2) \eta^{2}\right)}, \\
F \equiv & E-T S_{B H} \\
= & -\frac{V_{D-2}}{16 \pi G} \frac{8-(D-2) \eta^{2}}{8+(D-2) \eta^{2}} \\
& \times r_{h}^{\left(8(D-1)-(D-2) \eta^{2}\right) /\left(8+(D-2) \eta^{2}\right)} .
\end{aligned}
$$

Using the pressure defined by $P=-\partial F / \partial V_{D-2}$, the thermodynamic quantities of the EdBB satisfy the first law of thermodynamics as well as the Gibbs-Duhem relation, $E+$ $P V_{D-2}=T S_{B H}$. Following the gauge/gravity duality, these thermodynamic quantities can also be reinterpreted as those of the dual relativistic nonconformal theory.

\section{Holographic Renormalization of the Einstein-Dilaton Theory}

In the AdS/CFT correspondence, the conformal symmetry usually plays an important role to match spectra of gravity with their dual operators. In the nonconformal case, although such a relation is not clear, we can still investigate some thermodynamic properties of the dual field theory through the gauge/gravity duality. If the on-shell gravity action is identified with the free energy of the dual field theory, we can easily derive the thermodynamic properties. In this section, we will show that the on-shell gravity action, after the appropriate holographic renormalization, really provides the consistent thermodynamics with the EdBB thermodynamics.

In order to describe a finite temperature system, it is more convenient to take into account an Euclidean version. With an Euclidean signature, the gravitational action of the Einsteindilaton theory can be rewritten as

$$
S_{g r}=S_{E d}+S_{G H}
$$

with

$$
\begin{aligned}
S_{E d}= & -\frac{1}{16 \pi G} \\
& \times \int_{\mathscr{M}} d^{D} x \sqrt{g}\left[\mathscr{R}-2(\partial \phi)^{2}-2 \Lambda_{\mathrm{eff}} e^{\eta \phi}\right], \\
& S_{G H}=\frac{1}{8 \pi G} \int_{\partial \mathscr{M}} d^{D-1} x \sqrt{\gamma} \Theta,
\end{aligned}
$$

where $g_{\mu \nu}$ or $\gamma_{a b}$ is the Euclidean metric in the bulk or the induced boundary metric, respectively. For a well-defined action variation, the Gibbons-Hawking term $S_{G H}$ is required. An extrinsic curvature tensor $\Theta_{\mu \nu}$ is defined by

$$
\Theta_{\mu \nu}=-\frac{1}{2}\left(\nabla_{\mu} n_{\nu}+\nabla_{\nu} n_{\mu}\right)
$$

where $\nabla_{\mu}$ and $n_{\nu}$ mean a covariant derivative and an unit normal vector, respectively. Since the Gibbons-Hawking term is a boundary term, it does not affect the equations of motion.

Before evaluating the on-shell gravity action, it is worth noting that the on-shell gravity action usually suffers from the divergence when the boundary is located at $r=\infty$. To remove such a UV divergence (in [46, 47], the nonconformal field theory dual to a $p$-brane geometry in the dual frame has been investigated), we should add appropriate counter terms which make the on-shell gravity action become finite [49-51]. The correct counter term we found is

$$
S_{c t}=\frac{1}{8 \pi G} \int_{\partial \mathscr{M}} d^{D-1} x \sqrt{\gamma}\left(\frac{8(D-2)}{8+(D-2) \eta^{2}} e^{\eta \phi / 2}\right) .
$$

For $\eta=0$ it reduces, after restoring the AdS radius $R$, to the usual one for $\mathrm{AdS}_{D}$ space [49]

$$
S_{\text {AdS }}=\frac{1}{8 \pi G} \int_{\partial \mathscr{M}} d^{D-1} x \sqrt{\gamma} \frac{(D-2)}{R} .
$$

In general, there exist additional counter terms proportional to the boundary curvature scalar or tensors. However, since 
the boundary space of the EdBB geometry is flat with a Poincare symmetry $S O(1, D-2)$, the counter terms associated with the curvature scalar or tensors automatically vanish. Therefore, the resulting renormalized action can be described by

$$
S=S_{E d}+S_{G H}+S_{c t} .
$$

Since the on-shell gravity action reduces to a boundary term, it can be naturally interpreted as a boundary quantity. Following the strategy of the AdS/CFT correspondence, it should be proportional to the free energy of the dual theory with providing the same thermodynamics derived in the EdBB geometry. Now, let us check if the renormalized onshell gravity action really reproduces the results of the EdBB in (16).

First, consider the Einstein-dilaton action. Using the Einstein equation in (8), it simply reduces to

$$
\begin{aligned}
S_{E d}= & -\frac{1}{16 \pi G} \int_{0}^{\beta} d \tau \int d^{D-2} x \\
& \times \int_{r_{h}}^{r_{0}} d r \sqrt{g} \frac{4}{D-2} \Lambda_{\mathrm{eff}} e^{\eta \phi},
\end{aligned}
$$

where $\tau$ is an Euclidean time with a periodicity $\beta$ and $\int d^{D-2} x=V_{D-2}$ is the spatial volume of the boundary space. In the above, $r_{0}$ is introduced to denote the position of the boundary which can be interpreted as a UV cutoff of the dual theory. After inserting solutions (5) and (6) into (24) and evaluating it, we finally reach to

$$
S_{E d}=\frac{\beta V_{D-2}}{16 \pi G} \frac{16}{8+(D-2) \eta^{2}}\left(r_{0}^{c}-r_{h}^{c}\right),
$$

which diverges as $r_{0} \rightarrow \infty$ because of $c>1$. Similarly, the Gibbons-Hawking term and the counter term result in

$$
\begin{aligned}
S_{G B}= & -\frac{\beta V_{D-2}}{8 \pi G} \\
& \times\left(\frac{8(D-1)}{8+(D-2) \eta^{2}}-\frac{8(D-1)+(D-2) \eta^{2}}{2\left(8+(D-2) \eta^{2}\right)} \frac{r_{h}^{c}}{r_{0}^{c}}\right) r_{0}^{c}, \\
& S_{c t}=\frac{\beta V_{D-2}}{16 \pi G} \frac{8(D-2)}{8+(D-2) \eta^{2}} \sqrt{1-\frac{r_{h}^{c}}{r_{0}^{c}}} r_{0}^{c} .
\end{aligned}
$$

Summing all results, the exact renormalized action finally becomes

$$
\begin{gathered}
S=-\frac{\beta V_{D-2}}{16 \pi G}\left[\frac{16(D-2)}{8+(D-2) \eta^{2}}\left(1-\sqrt{1-\frac{r_{h}^{c}}{r_{0}^{c}}}\right) r_{0}^{c}\right. \\
\left.-\frac{8(D-3)+(D-2) \eta^{2}}{8+(D-2) \eta^{2}} r_{h}^{c}\right] .
\end{gathered}
$$

For $r_{0} \rightarrow \infty$, the renormalized on-shell action simply reduces to

$$
S=-\frac{\beta V_{D-2}}{16 \pi G} \frac{8-(D-2) \eta^{2}}{8+(D-2) \eta^{2}} r_{h}^{c} .
$$

Like the AdS/CFT correspondence, the free energy of the dual theory can be defined by $F=S / \beta$ which gives rise to the same free energy in (17).

Now, let us evaluate the boundary energy-momentum tensor. From the gravitational action in (18), the corresponding boundary stress tensor defined by $T_{a b}=-(2 / \sqrt{\gamma})$ $\left(\partial \mathcal{S} / \partial \gamma^{a b}\right)$ reads

$$
T_{(b d)}^{a}{ }_{b}=-\frac{1}{8 \pi G} \int d^{D-2} x \sqrt{\gamma} \gamma^{a c}\left(\Theta_{c b}-\gamma_{c b} \Theta\right),
$$

and the contribution from the counter term is

$$
T_{(c t)}{ }^{a}{ }_{b}=\frac{V_{D-2}}{8 \pi G} \frac{8(D-2)}{8+(D-2) \eta^{2}} \sqrt{\gamma} e^{\eta \phi / 2} \delta_{b}^{a},
$$

where the indices $a$ and $b$ imply the directions of the boundary space and time. So the renormalized stress tensor is given by the sum of them

$$
T_{b}^{a}=T_{(b d) b}^{a}+T_{(c t) b}^{a} .
$$

The explicit form of the renormalized boundary energy reads

$$
\begin{aligned}
E= & T_{\tau}^{\tau} \\
= & \frac{V_{D-2}}{8 \pi G} \frac{8(D-2)}{8+(D-2) \eta^{2}} \\
& \times\left[\sqrt{\left.1-\frac{r_{h}^{c}}{r_{0}^{c}}-\left(1-\frac{r_{h}^{c}}{r_{0}^{c}}\right)\right] r_{0}^{c},}\right.
\end{aligned}
$$

and the pressure becomes

$$
\begin{aligned}
& P=-\frac{T^{i}{ }_{i}}{V_{D-2}} \\
&=\frac{1}{8 \pi G}\left[\frac{8(D-2)}{8+(D-2) \eta^{2}}\left(1-\sqrt{1-\frac{r_{h}^{c}}{r_{0}^{c}}}\right)\right. \\
&\left.\quad-\frac{8(D-3)+(D-2) \eta^{2}}{2\left(8+(D-2) \eta^{2}\right)} \frac{r_{h}^{c}}{r_{0}^{c}}\right] r_{0}^{c},
\end{aligned}
$$

where the pressure is the same as $-F$. If we put a UV cutof into infinity $r_{0}=\infty$, the energy and pressure are simply reduced to

$$
\begin{aligned}
& E=\frac{V_{D-2}}{8 \pi G} \frac{4(D-2)}{8+(D-2) \eta^{2}} r_{h}^{c}, \\
& P=\frac{1}{16 \pi G} \frac{8-(D-2) \eta^{2}}{8+(D-2) \eta^{2}} r_{h}^{c} .
\end{aligned}
$$

These results show that the holographic renormalization of the Einstein-dilaton theory reproduces the exact same EdBB thermodynamics when the boundary is located at infinity.

At zero temperature $\left(r_{h}=0\right)$, the free energy and the internal energy of the dual theory become zero. In [33], it was shown that for $\eta^{2} \geq 8 /(D-2)$ the EdBB and its dual theory are thermodynamically unstable. In the parameter range 


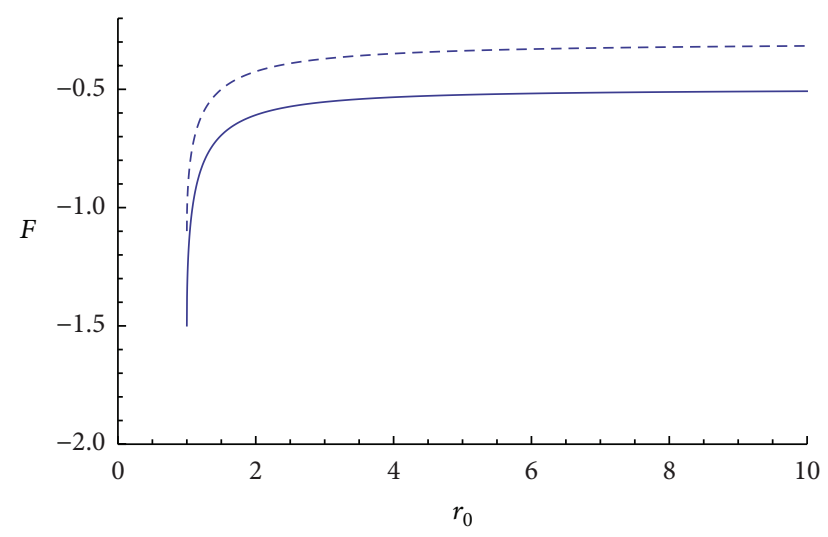

Figure 1: The free energy depending on $r_{0}$ for $\eta=0$ (solid) and $\eta=1$ (dashed), where we set $8 \pi G=1, r_{h}=1, D=4$, and $V_{2}=1$.

$\eta^{2}<8 /(D-2)$, the free energy at finite temperature is always negative. Therefore, if we do not insert an IR cutoff by hand, there is no Hawking-Page transition and the EdBB geometry is always preferable. This result is qualitatively the same as the one obtained in [52], where a different renormalization scheme, the so-called subtraction method, was used. Now, let us consider the UV cutoff dependence of the free energy. If we interpret the position of boundary as the energy scale of the dual theory, we can see how the free energy depends on the energy scale of the dual theory. In Figure 1, we draw the free energy depending on $r_{0}$ for $\eta=0$ and 1 , which shows that the free energy monotonically decreases as the energy scale of the dual theory decreases.

Following the definition of the equation of state parameter, it becomes in the dual relativistic nonconformal system

$$
w=\frac{1}{D-2}-\frac{\eta^{2}}{8}
$$

where the second term represents the deviation from the conformal one. This result also shows that the sound velocity of the relativistic nonconformal medium

$$
c_{s}=\sqrt{w}
$$

is always smaller than that of the relativistic conformal one, $\sqrt{1 /(D-2)}$.

\section{Membrane Paradigm}

In the previous section, the thermodynamic properties of a relativistic nonconformal system have been investigated by using the holographic renormalization. In this section, we will study the macroscopic properties, especially the hydrodynamic transport coefficients of the membrane paradigm [11], and compare them with the results of the Kubo formula $[33,52]$.

4.1. Charge Diffusion. In order to describe the charge diffusion process in the relativistic nonconformal medium, we need to introduce a Maxwell term describing $U(1)$ gauge field fluctuations on the EdBB. Due to the existence of the nontrivial dilaton field, the Maxwell term can have a more general gauge coupling depending on the radius $r$

$$
S=-\int d^{D} x \sqrt{-g} \frac{1}{4 g_{D}^{2}(r)} F_{M N} F^{M N}
$$

with

$$
g_{D}^{2}(r)=\frac{e^{\alpha \phi(r)}}{g_{0}^{2}}
$$

where $g_{0}^{2}$ is a constant and a new parameter $\alpha$ describes the strength of the gauge coupling. Usually, the vector fluctuations can be divided into two parts: if the fluctuation moves in the $y$-direction with a momentum $k$, one is the longitudinal modes, $A_{t}$ and $A_{y}$, and the other is the transverse modes $A_{x}$ (where $x$ means all transverse directions) in the $A_{r}=0$ gauge. Since the charge diffusion process is related to the motion of the longitudinal modes, we concentrate only on the longitudinal modes from now on. In the hydrodynamic limit $\left(\omega \sim k^{2}\right.$, $\omega \ll T_{H}$, and $k \ll T_{H}$ ), the Fourier mode expansions of the longitudinal modes become

$$
\begin{aligned}
& A_{t}(r, t, y)=\int \frac{d \omega d k}{(2 \pi)^{2}} e^{-i \omega t+i k y} A_{t}(r, \omega, k), \\
& A_{y}(r, t, y)=\int \frac{d \omega d k}{(2 \pi)^{2}} e^{-i \omega t+i k y} A_{y}(r, \omega, k) .
\end{aligned}
$$

These longitudinal modes satisfy two dynamical equations, the current conservation and the Bianchi identity. In terms of current $j^{\mu}$, the governing equations are [11]

$$
\begin{gathered}
0=-\partial_{r} j^{t}-\frac{\sqrt{-g}}{g_{D}^{2}(r)}\left(-g^{t t}\right) g^{y y} \partial_{y} F_{y t}, \\
0=-\partial_{r} j^{y}-\frac{\sqrt{-g}}{g_{D}^{2}(r)}\left(-g^{t t}\right) g^{y y} \partial_{t} F_{y t}, \\
0=\partial_{t} j^{t}+\partial_{y} j^{y}, \\
0=-\frac{g_{r r} g_{y y} g_{D}^{2}(r)}{\sqrt{-g}} \partial_{t} j^{y} \\
-\frac{g_{r r} g_{y y} g_{D}^{2}(r)}{\sqrt{-g}} \partial_{z} j^{t}+\partial_{r} F_{y t} .
\end{gathered}
$$

Combining these equations, one can easily derive a flow equation for the longitudinal conductivity defined by $\sigma_{L}\left(r, k_{\mu}\right) \equiv$ $j^{y} / F_{y t}$,

$$
\partial_{r} \sigma_{L}=i \omega \sqrt{\frac{g_{r r}}{\left(-g_{t t}\right)}}\left[\frac{\sigma_{L}^{2}}{\Sigma_{A}(r)}\left(1-\frac{k^{2}}{\omega^{2}} \frac{g^{y y}}{\left(-g^{t t}\right)}\right)-\Sigma_{A}(r)\right],
$$

with

$$
\Sigma_{A}(r)=\frac{1}{g_{D}^{2}(r)} \sqrt{\frac{-g}{\left(-g_{t t}\right) g_{r r}}} g^{y y}
$$


In the zero frequency limit, we can see that the longitudinal conductivity reduces to the DC conductivity and that it is independent of the position of the membrane. Furthermore, imposing the regularity of the conductivity at the horizon, the DC conductivity leads to

$$
\begin{aligned}
& \sigma_{D C} \\
& =\frac{1}{g_{0}^{2}}\left(\frac{4 \pi\left[8+(D-2) \eta^{2}\right]}{8(D-1)-(D-2) \eta^{2}}\right)^{(8(D-4)-2(D-2) \eta \alpha) /\left(8-(D-2) \eta^{2}\right)} \\
& \quad \times T_{H}^{(8(D-4)-2(D-2) \eta \alpha) /\left(8-(D-2) \eta^{2}\right)}
\end{aligned}
$$

which reproduces the Kubo formula's results obtained in specific parameter values, $\alpha=0$ and $\alpha=-\eta / 2$ [33]. From the general DC conductivity in (43), we can see that the DC conductivity of the relativistic nonconformal theory is always positive and real because the range of $\eta$ should be constrained to $\eta^{2}<8 /(D-2)$. Moreover, if $\alpha>4(D-4) /(D-2) \eta$ the DC conductivity decreases with increasing temperature. Taking $\alpha=\left(8(D-1)-(D-2) \eta^{2}\right) / 2(D-2) \eta$ for $D=5$ especially provides the resistivity proportional to temperature, which is the macroscopic electric property of the metal. If $\alpha=4(D-4) /(D-2) \eta$, the DC conductivity is independent of temperature. For $\alpha<4(D-4) /(D-2) \eta$, it increases with temperature, which is a typical feature of the electrolytes or some chemical compounds. In general, the macroscopic electric properties crucially depend on what the charge carriers are. For example, the electric property of the metal is mainly governed by the motion of electrons, whereas the motion of ions is important to understand the electric properties of the electrolytes or chemical compounds. As a result, we can say that parameter $\alpha$ provides the information for the charge carrier in the dual field theory.

In the hydrodynamic limit $\left(\omega, k \ll T_{H}\right)$, the flow equation of the longitudinal conductivity reduces to

$$
\frac{\partial_{r} \sigma_{L}}{\sigma_{L}^{2}}=-i \frac{k^{2}}{\omega} \frac{\left(-g_{t t}\right) g_{r r}}{\sqrt{-g}} g_{D}^{2}(r) .
$$

According to the holographic renormalization, the radial position of the membrane $r_{m}$ can be identified with the energy scale of the dual field theory. Using the fact that the DC conductivity plays a role of the initial data for the conductivity flow [11], integrating (44) from $r_{h}$ to $r_{m}$ gives rise to the following longitudinal conductivity:

$$
\sigma_{L}\left(r_{m}\right)=\frac{i \omega \sigma_{D C}}{i \omega-\mathscr{D}_{e}\left(r_{m}\right) k^{2}},
$$

where the charge diffusion constant $\mathscr{D}_{e}\left(r_{m}\right)$ depending on the energy scale is given by

$$
\begin{aligned}
\mathscr{D}_{e}\left(r_{m}\right) & =\sigma_{D C} \int_{r_{h}}^{r_{m}} d r \frac{\left(-g_{t t}\right) g_{r r}}{\sqrt{-g}} g_{D}^{2}(r) \\
& =\frac{\left(r_{h}^{-\delta}-r_{m}^{-\delta}\right)}{\delta} r_{h}^{\chi},
\end{aligned}
$$

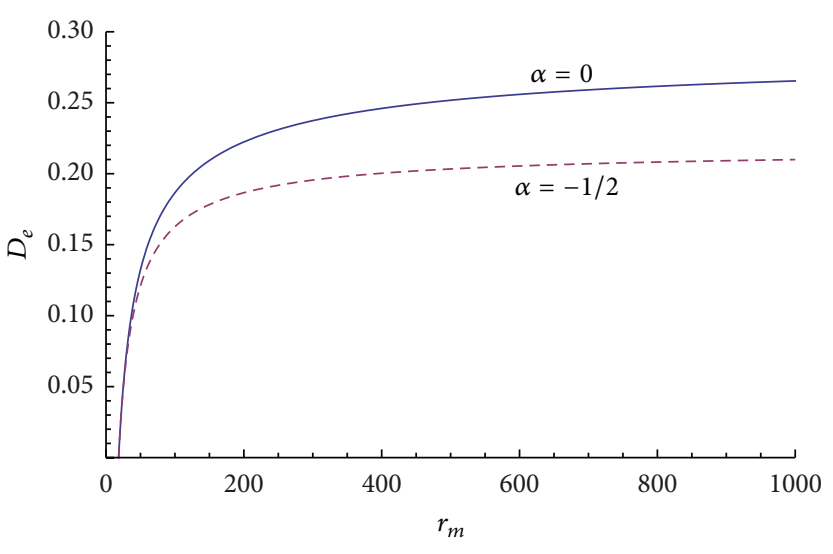

FIGURE 2: The RG flow of the charge diffusion constant, where we take $\eta=1, T_{H}=1$, and $D=4$. It shows that the charged diffusion constant increases monotonically with the energy scale denoted by $r_{m}$, and it becomes zero at the horizon $r_{h}=18.2521$.

with

$$
\begin{gathered}
\delta=\frac{8(D-3)-(D-2)(\eta+2 \alpha) \eta}{8+(D-2) \eta^{2}}, \\
\chi=\frac{8(D-4)-2(D-2) \alpha \eta}{8+(D-2) \eta^{2}} .
\end{gathered}
$$

For a positive $\delta\left(\right.$ or $\left.\alpha<\left(8(D-3)-(D-2) \eta^{2}\right) / 2(D-2) \eta\right)$, the charge diffusion constant of the dual field theory is welldefined in the limit of $r_{m} \rightarrow \infty$. Otherwise, it diverges at the infinity. For a welldefined hydrodynamic transport coefficients at the asymptotic boundary, we concentrate only on the positive $\delta$ from now on. Then, the general charge diffusion constant at the asymptotic boundary becomes

$$
\mathscr{D}_{e}(\infty)=\frac{1}{4 \pi T_{H}} \frac{8(D-1)-(D-2) \eta^{2}}{8(D-3)-(D-2)(\eta+2 \alpha) \eta} \text {. }
$$

In the 4-dimensional case, the charge diffusion constant at the asymptotic boundary is consistent with the result of the Kubo formula [33]. For more understanding, we take a special value of $\alpha$, for simplicity $\alpha=0$. Since $\eta$ is always smaller than $8 /(D-$ 2) due to the thermodynamic stability, the charge diffusion constant increases with $\eta$. This fact implies that the charge diffusion constant of the nonconformal medium is larger than that of the conformal one. Moreover, since the half-lifetime of the quasi-normal mode in the diffusion process is inversely proportional to the charge diffusion constant and the square of the momentum, the above result also implies that the quasi-normal mode decays more rapidly in the nonconformal medium. Lastly, we can easily see from (46) that the charge diffusion constant decreases monotonically as the energy of the dual theory runs from UV to IR (see Figure 2).

4.2. Momentum Diffusion. As shown in $[10,11]$, the relevant equations for gravitational shear modes $h_{t}^{x}$ and $h_{y}^{x}$ for $D=4$ can be mapped onto an electromagnetic problem by taking 
the analogy to the Kaluza-Klein reduction. If we set $h_{t}^{x}=a_{t}$ and $h_{y}^{x}=a_{y}$, the action for the shear modes reduces to

$$
S=\frac{1}{16 \pi} \int d^{D} x \sqrt{-g} g_{x x} F_{\alpha \beta} F^{\alpha \beta}
$$

where $\alpha$ and $\beta$ imply the longitudinal direction $t$ or $y$. Note that here $D \geq 4$; otherwise there is no shear mode. $F_{\alpha \beta}$ is the field strength of $a_{t}$ and $a_{y}$, which in terms of metric fluctuations is given by $F_{\alpha \beta}=\partial_{\alpha} h_{\beta}^{x}-\partial_{\beta} h_{\alpha}^{x}$ and easily generalized to the higher dimensional case. This action for shear modes is exactly the standard Maxwell form with an effective coupling $g_{G}^{2}$

$$
\frac{1}{g_{G}^{2}}=\frac{1}{16 \pi} g_{x x} .
$$

So we can immediately take over all results of the previous section.

The counterpart of the DC conductivity denoted by $\sigma_{G}$ becomes

$$
\sigma_{G}=\left.\frac{1}{16 \pi} \sqrt{\frac{-g}{\left(-g_{t t}\right) g_{r r}}}\right|_{r_{h}} .
$$

Notice that the Bekenstein-Hawking entropy density $s$ can be written as

$$
s \equiv \frac{S_{B H}}{V_{D-2}}=\left.\frac{1}{4} \sqrt{\frac{-g}{\left(-g_{t t}\right) g_{r r}}}\right|_{r_{h}} .
$$

From the celebrated universality in (2), one can easily see that $\sigma_{G}$ is nothing but the shear viscosity $\eta_{s}$ and that the result is the expected one in the Kubo formula [52].

Similarly, we can also easily evaluate the retarded Green function of the shear modes in the general $D$

$$
G_{R}^{x y, x y}=\frac{\eta_{s} \omega^{2}}{i \omega-D_{s}\left(r_{m}\right) k^{2}},
$$

where the momentum diffusion constant $\mathscr{D}_{s}\left(r_{m}\right)$, by taking the analogy to the charge diffusion constant, is

$$
\begin{aligned}
\mathscr{D}_{s}\left(r_{m}\right) & =4 s \int_{r_{h}}^{r_{m}} d r \frac{\left(-g_{t t}\right) g_{r r}}{\sqrt{-g} g_{x x}} \\
& =\frac{8+(D-2) \eta^{2}}{8(D-1)-(D-2) \eta^{2}}\left(\frac{1}{r_{h}^{\gamma}}-\frac{1}{r_{m}^{\gamma}}\right)
\end{aligned}
$$

with

$$
\gamma=\frac{8-(D-2) \eta^{2}}{8+(D-2) \eta^{2}}
$$

Below the crossover value $\eta^{2}<8 /(D-2)$ where the black brane is thermodynamically stable, $\gamma$ is always positive. If we put the membrane at the infinity and rewrite the momentum diffusion constant in terms of temperature, the momentum

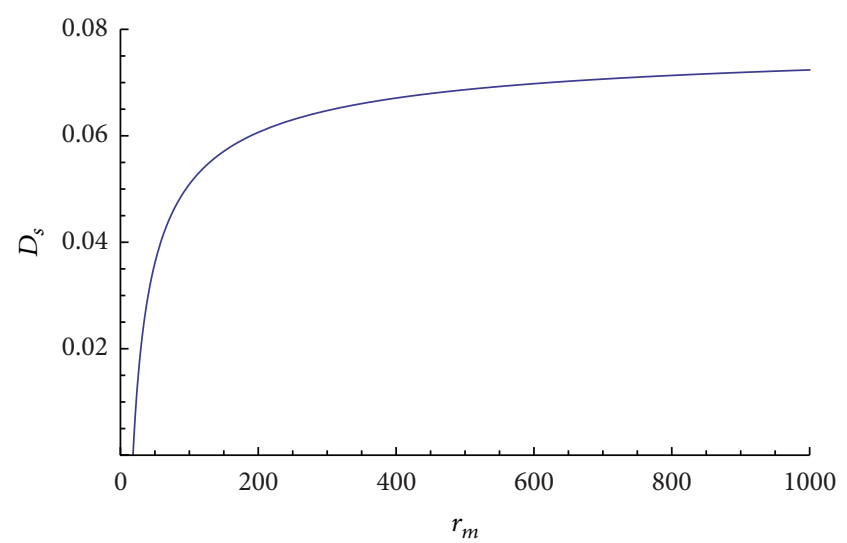

FIgURE 3: The RG flow of the momentum diffusion constant, where we take $\eta=1, T_{H}=1$, and $D=4$. Similarly to the charged diffusion constant, the momentum diffusion constant also increases monotonically with the energy scale $r_{m}$, and it becomes zero at the horizon $r_{h}=18.2521$.

diffusion constant has the form expected by the EdBB thermodynamics

$$
\mathscr{D}_{s}(\infty)=\frac{1}{4 \pi T_{H}},
$$

which shows the self-consistency of the gauge/gravity duality in the relativistic nonconformal medium. In addition, this result implies that the corresponding quasi-normal mode decays rapidly at high temperature, whereas the momentum diffusion constant does not depend on the nonconformality unlike the charge diffusion constant. Finally, we plot the momentum diffusion constant depending on the energy scale in Figure 3. It shows, similar to the charge diffusion constant, the monotonically decreasing behavior with decreasing energy.

\section{Binding Energies of Particles and Monopoles}

In the holographic QCD, the binding energy of quark and antiquark is described by a temporal Wilson loop, which in the string theory corresponds to the trajectory of the open string ends. In this section, we will investigate such a binding energy in the relativistic nonconformal medium. Before starting the calculation, it is worth to note that there is no difference between a fundamental string (or F1-string) and D1-brane (or D1-string) in the AdS space if we ignore the gravitational backreaction of the them. However, that is not true in the EdBB geometry due to the nontrivial dilaton profile. The metric usually felt by an open string is not in the Einstein frame but rather in the string frame represented as $[53,54]$

$$
d s_{\text {string }}^{2}=e^{\phi / 2} d s_{\text {Einstein }}^{2}
$$

which is the relation between frames of a 10-dimensional string theory. We can also introduce a dual frame [45-47] in 
which the near horizon geometry of $p$-branes is described by AdS $_{p+2} \times S^{8-p}$. Note that all these frames are related to each other by appropriate conformal mappings, and the geometry of p-brane in the string and Einstein frame can be generally expressed as the combination of a "warped AdS" and "warped sphere" geometry where "warped" means a proper multiplication of the dilaton field. In the Einstein frame, the EdBB geometry in (10) describes the noncompact geometry of a 10-dimensional string theory, and the other part, the compact geometry, can be described by an appropriate warped sphere. In investigations of the binding energy and drag force in the next section, since the internal space does not give any contribution, we can simply ignore it. Due to the nontrivial dilaton field of the EdBB geometry, the binding energies of particles and monopoles, which are represented by the ends of an F1- and D1-string, are different in the relativistic nonconformal theory. Therefore, the goal of this section is to investigate quantitatively the binding energies of particles and monopoles as well as to study the effect of the nonconformality on them.

In order to investigate the binding energy of two fundamental particles, we take into account the Nambu-Goto action of a fundamental string

$$
S_{F 1}=\frac{1}{2 \pi \alpha^{\prime}} \int d^{2} \sigma \sqrt{-\operatorname{det}\left(G_{\mu \nu} \frac{\partial x^{\mu}}{\partial \sigma^{\alpha}} \frac{\partial x^{\nu}}{\partial \sigma^{\beta}}\right)},
$$

where $G_{\mu \nu}$ is the space-time metric in the string frame. In the static gauge with the metric in (10)

$$
\begin{gathered}
\tau=t, \quad \sigma=x^{1}=x, \\
x^{2}=\cdots=x^{D-2}=0, \quad r=r(x),
\end{gathered}
$$

assuming that the end points of string are located at $\{r, x\}=$ $\{\infty, \pm l / 2\}$, the string action simply reduces to

$$
S_{F 1}=\frac{\beta}{2 \pi \alpha^{\prime}} \int_{-l / 2}^{l / 2} d x e^{\phi / 2} \sqrt{\dot{r}^{2}+r^{4 a_{1}} f(r)},
$$

where $\beta$ is the time interval and the dot means a derivative with respect to $x$. Using the analogy to mechanics, the conserved Hamiltonian after regarding $x$ as time is given by

$$
H=-\frac{e^{\phi / 2}}{2 \pi \alpha^{\prime}} \frac{r^{4 a_{1}} f(r)}{\sqrt{\dot{r}^{2}+r^{4 a_{1}} f(r)}} .
$$

If $r$ has a turning point or minimum value $r_{*}$ satisfying $\dot{r}_{*}=0$, the existence of such a turning point implies that the particle and antiparticle are connected by a string which corresponds to the bound state of particles. The absence of a turning point says that an open string connecting two particles divides into two straight strings describing free particles. First, we concentrate on the string configuration with a turning point at which the above conserved Hamiltonian is still satisfied

$$
H=-\frac{e^{\phi_{*} / 2}}{2 \pi \alpha^{\prime}} r_{*}^{2 a_{1}} \sqrt{f\left(r_{*}\right)},
$$

with $\phi_{*}=\phi\left(r_{*}\right)$.
After introducing a rescaled coordinate $\widetilde{r}=r / r_{*}$, comparing the above two Hamiltonians gives rise to information for the interdistance between particles in terms of the turning point

$$
l=\frac{2 \sqrt{f(1)}}{r_{*}^{2 a_{1}-1}} \int_{1}^{\infty} d \widetilde{r} \frac{1}{\widetilde{r}^{2 a_{1}} \sqrt{f(\widetilde{r})} \sqrt{e^{\widetilde{\phi}} \widetilde{r}^{4 a_{1}} f(\widetilde{r})-f(1)}},
$$

where $f(\widetilde{r})=1-\widetilde{r}_{h}^{c} / \widetilde{r}^{c}, f(1)=1-\widetilde{r}_{h}^{c}$, and $\widetilde{\phi}=\phi(\widetilde{r})$. In addition, an unrenormalized energy of a pair of particles becomes

$$
E \equiv \frac{S_{F 1}}{\beta}=\frac{r_{*}^{1-k_{0} / 2}}{\pi \alpha^{\prime}} \int_{1}^{\infty} d \widetilde{r} \frac{e^{\widetilde{\phi}} \widetilde{r}^{2 a_{1}} \sqrt{f(\widetilde{r})}}{\sqrt{e^{\tilde{\phi}} \widetilde{r}^{4 a_{1}} f(\widetilde{r})-f(1)}} .
$$

In the asymptotic region $(\widetilde{r} \rightarrow \infty)$, the unrenormalized energy has the following approximate form:

$$
E \approx \frac{r_{*}^{1-k_{0} / 2}}{\pi \alpha^{\prime}} \frac{\tilde{r}^{1-k_{0} / 2}}{1-k_{0} / 2} .
$$

Since $1-k_{0} / 2>0$ for $\eta^{2}<8 /(D-2)$, the unrenormalized energy diverges when $\widetilde{r} \rightarrow \infty$. In order to define the binding energy well, we need to renormalize it by adding an appropriate counter term. In the holographic QCD, this kind of divergence appears due to the infinite masses of two quarks described by straight strings. Therefore, we can remove the above divergence by subtracting the infinite particle masses. To do so, we parameterize two straight strings as

$$
\tau=t, \quad \sigma=r, \quad x^{1}= \pm \frac{l}{2} .
$$

Then, the energy of two straight strings is given by

$$
E_{c t}=\frac{r_{*}^{1-k_{0} / 2}}{\pi \alpha^{\prime}} \int_{\widetilde{r}_{h}}^{\infty} d \widetilde{r} e^{\widetilde{\phi} / 2},
$$

which corresponds to the mass of two particles and can exactly cancel the divergence of the unrenormalized energy. The renormalized energy of a pair of particles becomes

$$
\begin{aligned}
V & \equiv E-E_{c t} \\
& =\frac{r_{*}^{1-k_{0} / 2}}{\pi \alpha^{\prime}}\left(\int_{1}^{\infty} d \widetilde{r} \frac{e^{\widetilde{\phi}} r^{2 a_{1}} \sqrt{f(\widetilde{r})}}{\sqrt{e^{\tilde{\phi}} r^{4 a_{1}} f(\widetilde{r})-f(1)}}-\int_{\widetilde{r}_{h}}^{\infty} d \widetilde{r} e^{\tilde{\phi} / 2}\right),
\end{aligned}
$$

which corresponds to the well-defined binding energy between the particle and antiparticle.

Now, let us consider the low temperature case $\left(\widetilde{r}_{h}=\right.$ $\left.r_{h} / r_{*} \ll 1\right)$, in which the interdistance and the binding energy of a pair of particles have the following expansion forms:

$$
\begin{gathered}
l=\frac{1}{r_{*}^{2 a_{1}-1}}\left(A_{0}+A_{1} \frac{r_{h}^{c}}{r_{*}^{c}}+\cdots\right), \\
V=B_{0} r_{*}^{1-k_{0} / 2}+B_{1} r_{*}^{1-k_{0} / 2-c}+B_{2} r_{h}^{1-k_{0} / 2}+\cdots,
\end{gathered}
$$


where ellipsis means higher order corrections and

$$
\begin{aligned}
& A_{0}=\frac{2 \sqrt{\pi}}{2 a_{1}-1} \frac{\Gamma\left(1 / 2+\left(2 a_{1}-1\right) /\left(4 a_{1}-k_{0}\right)\right)}{\Gamma\left(\left(2 a_{1}-1\right) /\left(4 a_{1}-k_{0}\right)\right)}, \\
& A_{1}=\frac{2 \sqrt{\pi}}{4 a_{1}-k_{0}}\left[\frac{\Gamma\left(1 / 2+\left(2 a_{1}-1\right) /\left(4 a_{1}-k_{0}\right)\right)}{\Gamma\left(\left(2 a_{1}-1\right) /\left(4 a_{1}-k_{0}\right)\right)}\right. \\
& +\frac{2-2 c-k_{0}}{2\left(4 a_{1}-k_{0}\right)} \\
& \left.\times \frac{\Gamma\left(1 / 2+\left(2 a_{1}-1+c\right) /\left(4 a_{1}-k_{0}\right)\right)}{\Gamma\left(1+\left(2 a_{1}-1+c\right) /\left(4 a_{1}-k_{0}\right)\right)}\right], \\
& B_{0}=-\frac{2}{\left(2-k_{0}\right) \sqrt{\pi} \alpha^{\prime}} \\
& \times \frac{\Gamma\left(1 / 2+\left(2 a_{1}-1\right) /\left(4 a_{1}-k_{0}\right)\right)}{\Gamma\left(\left(2 a_{1}-1\right) /\left(4 a_{1}-k_{0}\right)\right)}, \\
& B_{1}=\frac{1}{\left(4 a_{1}-k_{0}\right) \sqrt{\pi} \alpha^{\prime}} \\
& \times\left[\frac{\Gamma\left(1 / 2+\left(2 a_{1}-1\right) /\left(4 a_{1}-k_{0}\right)\right)}{\Gamma\left(\left(2 a_{1}-1\right) /\left(4 a_{1}-k_{0}\right)\right)}\right. \\
& \left.-\frac{\Gamma\left(1 / 2+\left(2 a_{1}-1+c\right) /\left(4 a_{1}-k_{0}\right)\right)}{\Gamma\left(\left(2 a_{1}-1+c\right) /\left(4 a_{1}-k_{0}\right)\right)}\right], \\
& B_{2}=\frac{1}{\pi \alpha^{\prime}\left(1-k_{0} / 2\right)} \text {. }
\end{aligned}
$$

In the zero temperature limit $\left(r_{h} \rightarrow 0\right)$, the binding energy shows a Coulomb-like potential with a power depending on the nonconformality

$$
V=\frac{A_{0}^{\gamma} B_{0}}{l^{\gamma}}
$$

with

$$
\gamma=\frac{8-(D-2) \eta+(D-2) \eta^{2}}{8-(D-2) \eta^{2}} .
$$

For the conformal theory $(\eta=0)$ dual to the AdS space, the binding energy is given by the Coulomb potential proportional to $l^{-1}$, as expected by the conformality. For $0<\eta<$ $1 / 2, \gamma$ is positive and smaller than 1 . This implies that when compared with the conformal case the magnitude of the binding energy in the nonconformal medium slowly decreases as the interdistance of the two particles increases. Interestingly, the Coulomb potential inversely proportional to the interdistance again appears at $\eta=1 / 2$. Finally, for $1 / 2<\eta<$ $2 \sqrt{2} /(D-2)$ the binding energy is steeper than the one of the conformal case.

In order to investigate the thermal correction at low temperature, we need to rewrite $r_{*}$ in terms of $l$ and $r_{h}$. To do so, let us set

$$
r_{*}=\left(\frac{A_{0}}{l}\right)^{1 /\left(2 a_{1}-1\right)}(1+\delta) \text {, }
$$

where $\delta$ corresponds to the first thermal correction and is a function of $l$ and $r_{h}$. Since (74) should satisfy (69) at least at the first order of correction, $\delta$ must be

$$
\begin{aligned}
\delta= & \frac{1}{2 a_{1}-1} A_{0}^{-\left(2 a_{1}-1+c\right) /\left(2 a_{1}-1\right)} \\
& \times A_{1} l^{c /\left(2 a_{1}-1\right)} r_{h}^{c} .
\end{aligned}
$$

Inserting this result together with (74) into (70) gives rise to

$$
V=\frac{A_{0}^{\gamma} B_{0}}{l^{\gamma}}\left[1+K l^{c /\left(2 a_{1}-1\right)} r_{h}^{c}\right]
$$

where $K$ is given by

$$
\begin{aligned}
K= & \frac{2-k_{0}}{2\left(2 a_{1}-1\right)} A_{0}^{-\left(2 a_{1}-1+c\right) /\left(2 a_{1}-1\right)} A_{1} \\
& +\frac{B_{1}}{B_{0}} A_{0}^{-c /\left(2 a_{1}-1\right)},
\end{aligned}
$$

and $r_{h}^{c}$ is related to temperature

$$
\begin{aligned}
r_{h}^{c}= & \left(\frac{4 \pi\left\{8+(D-2) \eta^{2}\right\}}{8(D-1)+(D-2) \eta^{2}}\right)^{\left(8(D-1)-(D-2) \eta^{2}\right) /\left(8-(D-2) \eta^{2}\right)} \\
& \times T^{\left(8(D-1)-(D-2) \eta^{2}\right) / 8-(D-2) \eta^{2}} .
\end{aligned}
$$

As a result, the first thermal correction to the binding energy is proportional to

$$
\begin{aligned}
V_{T} \sim & l^{(D-2)\left(8+\eta-2 \eta^{2}\right) /\left(8-(D-2) \eta^{2}\right)} \\
& \times T^{\left(8(D-1)-(D-2) \eta^{2}\right) /\left(8-(D-2) \eta^{2}\right)} .
\end{aligned}
$$

This result shows that the thermal correction to the binding energy nontrivially depends on the interdistance and temperature with the power determined by the nonconformality and dimension. Especially, for the conformal case $\eta=0$ the first thermal correction is proportional to

$$
V_{T} \sim l^{D-2} T^{D-1} .
$$

At finite temperature, the binding energy of particles is calculated numerically in Figure 4. This result shows that the magnitude of the binding energy increases with increasing nonconformality $\eta$.

It is also possible to think of the binding energy for a pair of monopole and antimonopole by considering a $D 1$-string instead of an F1-string. In the dual theory, the end of D1string corresponds to a monopole, or anti-monopole whereas the end of a fundamental string describes a fundamental particle. In the AdS background, since there is no nontrivial dilaton profile, the binding energy of a pair of monopole and anti-monopole is the same as the one for particles. However, there exists a nontrivial dilaton field in the dual geometry of the relativistic nonconformal theory, so the binding energy is different from the F1-string result. In order to investigate 


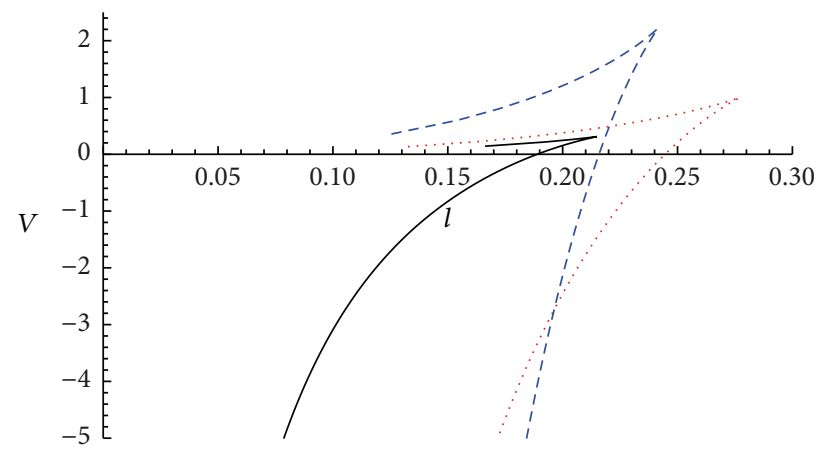

FIGURE 4: At given temperature $T=1$ the binding energy of particles for $\eta=0$ (solid), which is the same as the one of monopoles. The dashed or dotted one represents the binding energies of particles or monopoles for $\eta=1$, respectively, where we set $\pi \alpha^{\prime}=1$ and $D=4$.

the binding energy of a pair of monopole and anti-monopole, we should consider a Nambu-Goto action of a D1-string in the string frame, which contains an extra dilaton field contribution

$$
\begin{aligned}
S_{D 1} & =\frac{1}{2 \pi \alpha^{\prime}} \int d^{2} \sigma e^{-\phi} \sqrt{-\operatorname{det}\left(G_{\mu \nu} \frac{\partial x^{\mu}}{\partial \sigma^{\alpha}} \frac{\partial x^{\nu}}{\partial \sigma^{\beta}}\right)} \\
& =\frac{\beta}{2 \pi \alpha^{\prime}} \int_{-l / 2}^{l / 2} d x e^{-\phi / 2} \sqrt{\dot{r}^{2}+r^{4 a_{1}} f(r)},
\end{aligned}
$$

where the same parameterization (59) is used. In terms of the dimensionless coordinate $\widetilde{r}$, the interdistance of two monopoles can be rewritten as

$$
l=\frac{2 \sqrt{f(1)}}{r_{*}^{2 a_{1}-1}} \int_{1}^{\infty} d \widetilde{r} \frac{1}{\widetilde{r}^{2 a_{1}} \sqrt{f(\widetilde{r})} \sqrt{e^{-\widetilde{\phi}} \widetilde{r}^{4 a_{1}} f(\widetilde{r})-f(1)}}
$$

where $f(\widetilde{r})=1-\widetilde{r}_{h}^{c} / \widetilde{r}^{c}$ and $\widetilde{\phi}=\phi(\widetilde{r})$, and the unrenormalized energy is

$$
E=\frac{r_{*}^{1+k_{0} / 2}}{\pi \alpha^{\prime}} \int_{1}^{\infty} d \widetilde{r} \frac{e^{-\tilde{\phi}} \widetilde{r}^{2 a_{1}} \sqrt{f(\widetilde{r})}}{\sqrt{e^{-\tilde{\phi}} \widetilde{r}^{4 a_{1}} f(\tilde{r})-f(1)}} .
$$

Similar to the F1-string the divergence of the above unrenormalized energy can be renormalized by the following counter term, which corresponds to infinitely massive two monopoles described by two straight $D 1$-strings:

$$
E_{c t}=\frac{r_{*}^{1+k_{0} / 2}}{\pi \alpha^{\prime}} \int_{\widetilde{r}_{h}}^{\infty} d \widetilde{r} e^{-\widetilde{\phi} / 2}
$$

where the same parameterization in (66) is used. Then, the resulting renormalized binding energy of a pair of monopole and anti-monopole reduces to

$$
\begin{aligned}
V= & \frac{r_{*}^{1+k_{0} / 2}}{\pi \alpha^{\prime}} \\
& \times\left(\int_{1}^{\infty} d \widetilde{r} \frac{e^{-\widetilde{\phi}} r^{2 a_{1}} \sqrt{f(\widetilde{r})}}{\sqrt{e^{-\widetilde{\phi}} r^{4 a_{1}} f(\widetilde{r})-f(1)}}-\int_{\widetilde{r}_{h}}^{\infty} d \widetilde{r} e^{-\widetilde{\phi} / 2}\right) .
\end{aligned}
$$

At low temperature $\left(\widetilde{r}_{h}=r_{h} / r_{*} \ll 1\right)$, the interdistance and energy of a pair of monopoles have the following expansion forms:

$$
\begin{gathered}
l=\frac{1}{r_{*}^{2 a_{1}-1}}\left(C_{0}+C_{1} \frac{r_{h}^{c}}{r_{*}^{c}}+\cdots\right), \\
V=D_{0} r_{*}^{1+k_{0} / 2}+D_{1} r_{*}^{1+k_{0} / 2-c} r_{h}^{c} \\
+D_{2} r_{h}^{1+k_{0} / 2}+\cdots,
\end{gathered}
$$

where

$$
\begin{aligned}
& C_{0}= \frac{2 \sqrt{\pi}}{2 a_{1}-1} \frac{\Gamma\left(1 / 2+\left(2 a_{1}-1\right) /\left(4 a_{1}+k_{0}\right)\right)}{\Gamma\left(\left(2 a_{1}-1\right) /\left(4 a_{1}+k_{0}\right)\right)}, \\
& C_{1}=\frac{2 \sqrt{\pi}}{4 a_{1}+k_{0}} \\
& \times\left[\frac{\Gamma\left(1 / 2+\left(2 a_{1}-1\right) /\left(4 a_{1}+k_{0}\right)\right)}{\Gamma\left(\left(2 a_{1}-1\right) /\left(4 a_{1}+k_{0}\right)\right)}\right. \\
&+\frac{2-2 c+k_{0}}{2\left(4 a_{1}+k_{0}\right)} \\
&\left.\quad \times \frac{\Gamma\left(1 / 2+\left(2 a_{1}-1+c\right) /\left(4 a_{1}+k_{0}\right)\right)}{\Gamma\left(1+\left(2 a_{1}-1+c\right) /\left(4 a_{1}+k_{0}\right)\right)}\right], \\
& D_{0}=-\frac{2}{\left(2+k_{0}\right) \sqrt{\pi} \alpha^{\prime}} \\
& \times \frac{\Gamma\left(1 / 2+\left(2 a_{1}-1\right) /\left(4 a_{1}+k_{0}\right)\right)}{\Gamma\left(\left(2 a_{1}-1\right) /\left(4 a_{1}+k_{0}\right)\right)}, \\
& D_{1}=\frac{1}{\left(4 a_{1}+k_{0}\right) \sqrt{\pi} \alpha^{\prime}} \\
& \times\left[\frac{\Gamma\left(1 / 2+\left(2 a_{1}-1\right) /\left(4 a_{1}+k_{0}\right)\right)}{\Gamma\left(\left(2 a_{1}-1\right) /\left(4 a_{1}+k_{0}\right)\right)}\right. \\
&\left.\quad-\frac{\Gamma\left(1 / 2+\left(2 a_{1}-1+c\right) /\left(4 a_{1}+k_{0}\right)\right)}{\Gamma\left(\left(2 a_{1}-1+c\right) /\left(4 a_{1}+k_{0}\right)\right)}\right], \\
& D_{2}=\frac{1}{\pi \alpha^{\prime}\left(1+k_{0} / 2\right)} .
\end{aligned}
$$


Similar to (74), $r_{*}$ can be rewritten in terms of $l$ and $r_{h}$ as

$$
\begin{aligned}
r_{*}= & \left(\frac{C_{0}}{l}\right)^{1 /\left(2 a_{1}-1\right)} \\
& \times\left(1+\frac{1}{2 a_{1}-1} C_{0}^{-\left(2 a_{1}-1+c\right) /\left(2 a_{1}-1\right)} C_{1} l^{c /\left(2 a_{1}-1\right)} r_{h}^{c}\right) .
\end{aligned}
$$

Inserting this result into the binding energy, we finally obtain

$$
V=\frac{A_{0}^{\chi} B_{0}}{l \chi}\left[1+L l^{c /\left(2 a_{1}-1\right)} r_{h}^{c}\right]
$$

where $\chi$ and $L$ are given by

$$
\begin{aligned}
\chi & =\frac{8+(D-2) \eta+(D-2) \eta^{2}}{8-(D-2) \eta^{2}} \\
L= & \frac{2+k_{0}}{2\left(2 a_{1}-1\right)} C_{0}^{-\left(2 a_{1}-1+c\right) /\left(2 a_{1}-1\right)} C_{1} \\
& +\frac{D_{1}}{D_{0}} C_{0}^{-c /\left(2 a_{1}-1\right)} .
\end{aligned}
$$

As a result, the first thermal correction is proportional to

$$
V_{T} \sim(l T)^{\left(8(D-1)-(D-2) \eta^{2}\right) /\left(8-(D-2) \eta^{2}\right)} .
$$

Unlike the binding energy of particles, the first thermal correction of monopoles has the same dependence on the interdistance and temperature. Similar to the particle case, the magnitude of the binding energy of monopoles at finite temperature increases as the nonconformality increases. From Figure 4, we see that a bound state of particles and monopoles in the conformal field theory can be dissociated more easily than in the nonconformal field theory. In addition, in the dual nonconformal field theory of the EdBB geometry, the dissociation length of a particle bound state is shorter than that of a monopole bound state due to the different coupling strength. This fact implies that a particle bound state, compared to a monopole bound state, can be easily dissociated to two free particles.

\section{Drag Force}

In this section, we will investigate the drag force of an external particle and a monopole in the nonconformal medium. As mentioned before, the action describing the motion of an F1or D1-string is defined in the string frame [55]. In the Einstein-dilaton theory, due to the nontrivial dilaton profile the Nambu-Goto action has an additional contribution from the dilaton field unlike the AdS case. In the static gauge

$$
\tau=t, \quad \sigma=r, \quad x_{1}=v t+x(r)
$$

the Nambu-Goto action reduces to

$$
S=\frac{1}{2 \pi \alpha^{\prime}} \int d^{2} \sigma e^{\zeta \phi / 2} \sqrt{1-\frac{v^{2}}{f}+r^{4 a_{1}} f x^{\prime 2}}
$$

where the prime means that the derivative with respect to $r$ and $\zeta$ is +1 or -1 for an $F 1$ - or D1-string, respectively, (In $[56,57]$, the drag force in the general hyperscaling violation background was investigated with $\zeta=0$ ). The conserved quantity, when regarding $r$ as a time, is represented as

$$
\Pi_{x}=e^{\zeta \phi / 2} \frac{r^{4 a_{1}} f x^{\prime}}{\sqrt{1-v^{2} / f+r^{4 a_{1}} f x^{\prime 2}}},
$$

where we set $2 \pi \alpha^{\prime}=1$. Rewriting $x^{\prime}$ as a function of $\Pi_{x}$ gives rise to

$$
x^{\prime}=\frac{\Pi_{x}}{r^{4 a_{1}} f} \sqrt{\frac{f-v^{2}}{e^{\zeta \phi} f-\Pi_{x}^{2} / r^{4 a_{1}}}} .
$$

For a well-defined $x^{\prime}$, the inside of the square root should be always positive. However, there exists a point $r_{s}$ at which $f\left(r_{s}\right)=v^{2}$ is saturated, so the denominator should also change its sign at that point. In terms of $r_{h}, r_{s}$ becomes

$$
r_{s}=\frac{r_{h}}{\left(1-v^{2}\right)^{1 / c}}
$$

and the conserved momentum is expressed by

$$
\Pi_{x}=\frac{v}{\left(1-v^{2}\right)^{\delta / c}} r_{h}^{\delta / 2},
$$

where $\delta=4 a_{1}-\zeta k_{0}$. [55]

The momentum flow along the string is represented by

$$
\Delta P_{1}=\int d t \sqrt{-g} P_{x^{1}}^{r}=\frac{d p_{1}}{d t} \Delta t,
$$

where the worldsheet current $P^{\alpha}{ }_{\mu}$ carried by a string with a nontrivial dilaton field is given by

$$
P_{\mu}^{\alpha}=-e^{\zeta \phi / 2} G_{\mu \nu} \partial^{\alpha} x^{\nu}
$$

Then, the drag force $d p_{1} / d t$ reads

$$
\frac{d p_{1}}{d t}=-\sqrt{-g} e^{\zeta \phi / 2} G_{x^{1} \nu} g^{r \alpha} \partial_{\alpha} x^{\nu}
$$

where $G_{x^{1} v}$ is the metric of the target space time and $g^{r \alpha}$ is the inverse of the induced worldsheet metric. Using (96) and (97), the drag force becomes

$$
\frac{d p_{1}}{d t}=-\frac{r_{h}^{\delta / 2} v}{\left(1-v^{2}\right)^{\delta / 2 c}},
$$

where the integral (98) is evaluated at the asymptotic boundary. This result guarantees that the momentum decreases as time evolves. In particular, for the $\mathrm{AdS}_{5}$ space, where, $\eta=\zeta=$ 0 it reproduces the result of [55]. Rewriting the above result in terms of physical quantities leads to

$$
\frac{d p_{1}}{d t}=-F\left(T_{H}\right) \frac{p_{1}\left(m^{2}+p_{1}^{2}\right)^{(\delta-c) / 2 c}}{m^{\delta / c}},
$$


with

$$
\begin{aligned}
& F\left(T_{H}\right) \\
& =\left(\frac{4 \pi\left(8+(D-2) \eta^{2}\right)}{8(D-1)+(D-2) \eta^{2}}\right)^{(16-(D-2) \zeta \eta) /\left(8-(D-2) \eta^{2}\right)} \\
& \quad \times T_{H}^{(16-(D-2) \zeta \eta) /\left(8-(D-2) \eta^{2}\right)},
\end{aligned}
$$

where $m$ and $p_{1}$ are the mass and the momentum of a particle or monopole depending on the value of $\zeta$.

In the nonrelativisitic limit $\left(m \gg p_{1}\right)$, the momentum decreases exponentially

$$
p_{1}(t)=p_{1}\left(t_{0}\right) e^{-\left(F\left(T_{H}\right) / m\right) t},
$$

where $p_{1}\left(t_{0}\right)$ is the momentum at $t=t_{0}$. The above result also shows that the drag force of a monopole $(\zeta=-1)$ increases more rapidly than the one of the particle $(\zeta=+1)$ as temperature increases. In the relativistic case $\left(m \ll p_{1}\right)$, the momentum decreases with power law behavior as time evolves

$$
p_{1}(t)=\left[p_{0}^{(c-\delta) / c}-\frac{c-\delta}{c} \frac{F\left(T_{H}\right)}{m^{\delta / c}} t\right]^{c /(c-\delta)},
$$

where $p_{0}$ expresses the momentum at $t_{1}=0$. For more understanding, if $c /(c-\delta)$ is rewritten in terms of intrinsic parameters as

$$
\frac{c}{c-\delta}=\frac{8(D-1)-(D-2) \eta^{2}}{8(D-5)+2(D-2) \zeta \eta-(D-2) \eta^{2}} .
$$

it reduces to

$$
\begin{gathered}
\frac{c}{c-\delta}=\frac{24-2 \eta^{2}}{-8+4 \zeta \eta-2 \eta^{2}} \quad \text { for } D=4, \\
\frac{c}{c-\delta}=\frac{32-3 \eta^{2}}{-6 \zeta \eta+3 \eta^{2}} \quad \text { for } D=5 .
\end{gathered}
$$

Assuming that $\eta$ is positive, the thermodynamically stable parameter range of $\eta$ for $D=4$ is given by $0 \leq \eta<2$. In this parameter range, $c /(c-\delta)$ is always negative regardless of $\zeta$. Therefore, the momenta of a particle and a monopole decrease as the inverse power of time

$$
p_{1}(t)=\frac{1}{\left[1 / p_{0}^{\gamma}+\left(F\left(T_{H}\right) / \gamma m^{\delta / c}\right) t\right]^{\gamma}},
$$

where $\gamma=c /(\delta-c)>0$. In the thermodynamically stable parameter range for $D=5, c /(c-\delta)$ is always negative for a particle and positive for a monopole. So the momentum of a monopole gives rise to

$$
p_{1}(t)=\left[p_{0}^{(c-\delta) / c}-\frac{c-\delta}{c} \frac{F\left(T_{H}\right)}{m^{\delta / c}} t\right]^{c /(c-\delta)},
$$

while a particle shows the inverse power law behavior in (108). In all cases, the momentum decreases more rapidly at high temperature. In sum, the drag force calculation shows that the momentum of a particle and monopole is suppressed exponentially for the nonrelativistic case and in a power law for the relativistic case. In the dual nonconformal theory of the EdBB geometry, the suppression powers of a particle and monopole are different and crucially depend on the nonconformality while there is no difference in the conformal field theory dual to the AdS geometry.

\section{Holographic Entanglement Entropy}

Recently, there was an interesting conjecture that in a small subsystem the entanglement temperature has a universal feature proportional to the inverse of size $l[34]$

$$
T_{\text {en }} \sim \frac{1}{l} .
$$

In this section, we will check such a universal feature in the relativistic nonconformal theory. Before doing that, we first check whether the EdBB can provide a consistent dual geometry or not following [41]. To do so, it is more convenient to rewrite the metric as the hyperscaling violation form. After introducing

$$
\begin{gathered}
r \longrightarrow\left(2 a_{1}-1\right)^{1 /\left(1-a_{1}\right)} u^{-1 /\left(2 a_{1}-1\right)}, \\
\left\{t, x^{i}\right\} \longrightarrow\left(2 a_{1}-1\right)^{-a_{1} /\left(1-a_{1}\right)}\left\{t, x^{i}\right\},
\end{gathered}
$$

the metric becomes

$$
\begin{aligned}
d s^{2}= & u^{-2(D-2-\theta) /(D-2)} \\
& \times\left(-f(u) d t^{2}+\frac{d u^{2}}{f(u)}+\delta_{i j} d x^{i} d x^{j}\right),
\end{aligned}
$$

where the black brane factor $f(u)$ is

$$
f(u)=1-\left(\frac{u}{u_{h}}\right)^{c /\left(2 a_{1}-1\right)} .
$$

Here, the hyperscaling violation exponent $\theta$ is given by

$$
\theta=-\frac{(D-2)^{2} \eta^{2}}{8-(D-2) \eta^{2}} .
$$

Now, let us concentrate on the symmetry of the asymptotic geometry. Since the boundary is located at $u=0$ in the new coordinate, the asymptotic metric reduces to

$$
\begin{aligned}
d s^{2}= & u^{-2(D-2-\theta) /(D-2)} \\
& \times\left(-d t^{2}+d u^{2}+\delta_{i j} d x^{i} d x^{j}\right) .
\end{aligned}
$$

Under the following scaling transformation

$$
t \longrightarrow \lambda t, \quad u \longrightarrow \lambda u, \quad x^{i} \longrightarrow \lambda x^{i},
$$

the metric transforms as $d s^{2} \rightarrow \lambda^{2 \theta /(D-2)} d s^{2}$, in which the nonzero value of $\theta$ indicates the breaking of the conformal 
symmetry of the dual field theory. Nevertheless, the rotational and translational symmetries of the boundary space represent that the dual theory is still relativistic. As a result, the dual theory of the EdBB geometry maps on to a relativistic nonconformal field theory. In this case, the null energy condition reads

$$
\theta[(D-2)-\theta] \leq 0
$$

For a consistent gravity dual, $\theta$ should satisfy this null energy condition [41]. Since $\theta$ in (114) satisfies the null energy condition for all ranges of $\eta$

$$
\begin{gathered}
\theta \leq 0 \text { for } \eta^{2}<\frac{8}{(D-2)}, \\
\theta \geq D-2 \text { for } \eta^{2}>\frac{8}{(D-2)},
\end{gathered}
$$

the EdBB geometry (115) is a consistent gravity dual of a relativistic nonconformal field theory. Although the entire ranges of $\eta$ provide a consistent gravity dual, only the range $\eta^{2}<$ $8 /(D-2)$ is thermodynamically stable.

Now, let us study the holographic entanglement entropy of such a relativistic nonconformal theory by using a $D-2$ dimensional strip $[58,59]$. A strip in the EdBB background can be parameterized by

$$
\begin{gathered}
-\frac{l}{2} \leq x^{1} \leq \frac{l}{2}, \\
0 \leq x^{i} \leq L \quad(i=2,3, \ldots, D-2),
\end{gathered}
$$

where $L$ corresponds to the interval of $x^{i}$ and we assume that $l \ll L$. Since the strip is extended in the radial direction $u$, its profile can be represented as a function of $x^{1}, u=u\left(x^{1}\right)$, with the following boundary conditions:

$$
\epsilon=u\left(-\frac{l}{2}\right)=u\left(\frac{l}{2}\right),
$$

where $\epsilon$ is an appropriate UV cutoff of the radial coordinate. The area of the strip then becomes

$$
A=2 L^{D-3} \int_{0}^{l / 2} d x^{1} u^{-(D-2-\theta)} \sqrt{\frac{u^{\prime 2}}{f(u)}+1},
$$

where the prime implies a derivative with respect to $x^{1}$. If $x^{1}$ is regarded as time, the conserved energy density is given by

$$
H=-\frac{2}{u^{D-2-\theta} \sqrt{u^{\prime 2} / f(u)+1}} .
$$

Now, let us assume that the strip configuration has a maximum value, $u_{\max }$, which corresponds to the turning point or tip of the U-shape configuration in the $x^{1}-u$ plane. At the turning point, since $u^{\prime}$ vanishes, the conserved energy density reduces to

$$
H=-\frac{2}{u_{\max }^{D-2-\theta}} .
$$

Comparing two conserved energy densities we can represent distance $l$ and area $A$ of strip in terms of $u_{\max }$

$$
\begin{gathered}
l=2 u_{\max } \int_{\epsilon}^{1} \frac{\tilde{u}^{D-2-\theta} d \tilde{u}}{\sqrt{f(\widetilde{u})} \sqrt{1-\widetilde{u}^{2(D-2-\theta)}}}, \\
A=-\frac{2 L^{D-3}}{u_{\max }^{D-3-\theta}} \int_{\epsilon}^{1} \frac{d \tilde{u}}{\tilde{u}^{D-2-\theta} \sqrt{f(\widetilde{u})} \sqrt{1-\widetilde{u}^{2(D-2-\theta)}}},
\end{gathered}
$$

where the new coordinate $\widetilde{u}$ is defined as $\widetilde{u}=u / u_{\max }$ and the black brane factor is in terms of $\widetilde{u}$

$$
f(\widetilde{u})=1-\left(\frac{\tilde{u}}{\tilde{u}_{h}}\right)^{c /\left(2 a_{1}-1\right)},
$$

with $\tilde{u}_{h}=u_{h} / u_{\max }$. It should be noted that the above U-shape configuration is only possible when $u_{\max }$ is smaller than $u_{h}$; in other words, $\widetilde{u}_{h}>1$. If not, the turning point of the strip goes inside of the black brane horizon. In this case, the resulting string configuration is described by two disconnected planes outside of the black brane horizon.

At zero temperature, $\tilde{u}_{h} \rightarrow \infty$ and $f(\widetilde{u}) \rightarrow 1$, respectively. So distance $l$ and the area of the strip $A$ simply reduce to

$$
\begin{gathered}
l=g_{0} u_{\max }, \\
A=\frac{\widetilde{h}_{0}}{u_{\max }^{D-3-\theta}},
\end{gathered}
$$

with

$$
\begin{gathered}
g_{0}=\frac{2 \sqrt{\pi} \Gamma((D-1-\theta) / 2(D-2-\theta))}{\Gamma(1 / 2(D-2-\theta))}, \\
\tilde{h}_{0}=-\frac{2 L^{D-3}}{(D-3-\theta)} \frac{1}{\epsilon^{D-3-\theta}}+h_{0}, \\
h_{0}=\frac{2 L^{D-3}}{(D-3-\theta)} \\
\times \frac{\sqrt{\pi} \Gamma((D-1-\theta) / 2(D-2-\theta))}{\Gamma(1 / 2(D-2-\theta))},
\end{gathered}
$$

where the first term in (129) represents a UV divergence as $\epsilon \rightarrow 0$. Ignoring the UV divergence, the zero temperature entanglement entropy reduces to

$$
\begin{aligned}
& S_{e n} \\
& =\frac{L^{D-3}\left[8-(D-2) \eta^{2}\right]}{2 G\left[8(D-3)+(D-2) \eta^{2}\right]} \\
& \quad \times\left(\frac{2}{l}\right)^{\left(8(D-3)+(D-2) \eta^{2}\right) /\left(8-(D-2) \eta^{2}\right)} \\
& \quad \times\left[\frac{\sqrt{\pi} \Gamma\left(\left(8(D-1)-(D-2) \eta^{2}\right) / 16(D-2)\right)}{\Gamma\left(\left(8-(D-2) \eta^{2}\right) / 16(D-2)\right)}\right]^{8(D-2) /\left(8-(D-2) \eta^{2}\right)} .
\end{aligned}
$$


If the size of the subsystem is small $\left(\widetilde{u}_{h} \gg 1\right)$, after removing the UV divergence, the distance and area of strip can be expanded into

$$
\begin{aligned}
& l=u_{\max }\left[g_{0}+g_{1}\left(\frac{u_{\max }}{u_{h}}\right)^{\zeta}\right]+\cdots, \\
& A=\frac{1}{u_{\max }^{\xi}}\left[h_{0}+h_{1}\left(\frac{u_{\max }}{u_{h}}\right)^{\zeta}\right]+\cdots,
\end{aligned}
$$

where

$$
\begin{gathered}
\zeta=\frac{8(D-1)-(D-2) \eta^{2}}{8-(D-2) \eta^{2}}, \\
\xi=\frac{8(D-3)+(D-2) \eta^{2}}{8-(D-2) \eta^{2}},
\end{gathered}
$$

$g_{1}$

$$
\begin{gathered}
=\frac{\sqrt{\pi}\left[8-(D-2) \eta^{2}\right] \Gamma\left(\left(8(2 D-3)-(D-2) \eta^{2}\right) / 8(D-2)\right)}{2\left[8(D-1)-(D-2) \eta^{2}\right] \Gamma\left(\left(4(3 D-4)-(D-2) \eta^{2}\right) / 8(D-2)\right)}, \\
h_{1}=-\frac{L^{D-3} \sqrt{\pi} \Gamma\left(\left(8(D-1)-(D-2) \eta^{2}\right) / 8(D-2)\right)}{2 \Gamma\left(\left(4 D-(D-2) \eta^{2}\right) / 8(D-2)\right)} .
\end{gathered}
$$

In order to describe the entanglement entropy in terms of the system size, we need to rewrite $u_{\max }$ in terms of $l$ and $u_{h}$. To do so, we first set $u_{\max }$ to

$$
u_{\max }=\frac{l}{g_{0}}(1+\delta),
$$

where $\delta$ is a small function of $l$ and $u_{h}$. Then, the first term satisfies the zero temperature result in (126), whereas the second corresponds to the leading thermal correction. In order to satisfy (132) at least at order of $u_{h}^{-\zeta}, \delta$ should be

$$
\delta=-\frac{g_{1}}{g_{0}^{\zeta+1}} \frac{l^{\zeta}}{u_{h}^{\zeta}} .
$$

When substituting this result into (133), the area of strip becomes up to order of $u_{h}^{-\zeta}$

$$
A=\frac{g_{0}^{\xi} h_{0}}{l^{\xi}}\left(1+M \frac{l^{\zeta}}{u_{h}^{\zeta}}\right),
$$

where $M$ is given by

$$
M=\frac{\xi g_{1}}{g_{0}^{\zeta+1}}+\frac{h_{1}}{h_{0} g_{0}^{\zeta}} .
$$

Using the following relation together with (136)

$$
\begin{aligned}
u_{h}= & \frac{8(D-1)+(D-2) \eta^{2}}{4 \pi\left(8+(D-2) \eta^{2}\right)} \\
& \times\left(\frac{8-(D-2) \eta^{2}}{8+(D-2) \eta^{2}}\right)^{\left(8-(D-2) \eta^{2}\right) /(D-2) \eta^{2}} \frac{1}{T_{H}},
\end{aligned}
$$

the entanglement entropy in the small size limit leads to

$$
S_{e n} \equiv \frac{A}{4 G}=\frac{g_{0}^{\xi} h_{0}}{4 G}\left(l^{-\xi}+\frac{M}{N^{c}} l^{\zeta-\xi} T_{H}^{\zeta}\right),
$$

where the first term represents the entanglement entropy at zero temperature and the second is the leading thermal correction. In other words, the leading temperature-dependent entanglement entropy is proportional to

$$
\Delta S_{e n} \sim l^{2} T_{H}^{\left(8(D-1)-(D-2) \eta^{2}\right) /\left(8-(D-2) \eta^{2}\right)},
$$

which corresponds to the entropy increase caused by the excited states. Using the fact that the boundary energy density in (34) is proportional to $r_{h}^{c}$ only, we can easily evaluate the total energy of strip when the strip distant $l$ in (119) is very small

$$
\begin{aligned}
\Delta E & =\int d^{D-2} x \frac{1}{8 \pi G} \frac{4(D-2)}{8+(D-2) \eta^{2}} r_{h}^{c} \\
& \sim l T_{H}^{\left(8(D-1)-(D-2) \eta^{2}\right) /\left(8-(D-2) \eta^{2}\right)} .
\end{aligned}
$$

These results show that the leading temperature-dependent entanglement entropy and the energy of the excited states depend nontrivially on the Hawking temperature $T$, which is the temperature of the thermal equilibrium. However, the ratio of them is independent of the Hawking temperature

$$
\frac{\Delta S_{e n}}{\Delta E} \sim l
$$

which shows the universal feature conjectured in [34]. Introducing an entanglement temperature inversely proportional to distance $l$, the first law of thermodynamics is satisfied even in the relativistic nonconformal theory.

\section{Discussion}

In the $D$-dimensional Einstein-dilaton theory with a Liouville potential, a Schwarzschild-type black brane solution is allowed and its asymptotic geometry has the $\operatorname{ISO}(1, D-2)$ symmetry group. Following the gauge/gravity duality, this isometry group can be reinterpreted as the Poincare group of the dual theory defined on the boundary. In this case, because there is no scaling symmetry the dual theory becomes a relativistic nonconformal theory. In this paper, we have investigated the thermodynamic properties of the relativistic nonconformal theory by using the holographic renormalization. After introducing a correct counter term, we have evaluated the finite boundary stress tensor and showed that the thermodynamics derived from it coincides with that of the EdBB geometry. Furthermore we showed, after identifying the radial coordinate with the energy scale of the dual theory, that the free energy of the relativistic nonconformal theory monotonically decrease along the change of the energy from UV to IR.

For checking the self-consistency of the gauge/gravity duality in the Einstein-dilaton theory, we studied the hydrodynamics of the dual theory by using the membrane 
paradigm. The resulting transport coefficients coincide with those obtained by the Kubo formula. Furthermore, the thermodynamic quantity, $\epsilon+P$, read off from the momentum diffusion constant is also consistent with the QFT result. The charge and momentum diffusion constants monotonically decreases as the energy of the dual theory decreases.

Although the microscopic theory dual to the EdBB and the exact map between the bulk fluctuations and their dual operators are still unclear for the non-AdS geometry, our work shows the possibility to generalize the AdS/CFT correspondence to the non-AdS space. Based on such self-consistencies of the thermodynamic and macroscopic properties, we further investigate some physical properties of the relativistic nonconformal theory, the binding energies of particles and monopoles, and the drag forces of them. Due to the nontrivial coupling constant described by the dilaton, the particle and monopole have different physical properties in the nonconformal medium, whereas they are indistinguishable in the conformal theory. For example, for $\eta=1$ the binding energy of monopoles is stronger than that of particles. When the motion of the particle and monopole is nonrelativistic in the 4-dimensional relativistic nonconformal medium, the momentum of a particle dissipates with a power law while a monopole has the dissipation with an inverse power law. For a nonrelativistic particle and monopole, the momentum dissipates exponentially. In all cases, the dissipation rate is given by a function of the nonconformality. We lastly showed that the universal feature of the entanglement temperature also appears in the relativistic nonconformal theory.

The gauge/gravity duality in the non-AdS space is one of the important issues, so it remains interesting to investigate the microscopic aspects of it and to apply it to the real physical systems. We hope to report more results in the future works.

\section{Acknowledgments}

This work was supported by the Korea Science and Engineering Foundation (KOSEF) grant funded by the Korea government (MEST) through the Center for Quantum Spacetime (CQUeST) of Sogang University with Grant no. R11-2005-021. C. Park was also supported by the Basic Science Research Program through the National Research Foundation of Korea (NRF) funded by the Ministry of Education, Science and Technology (2010-0022369).

\section{References}

[1] J. Maldacena, "The large $N$ limit of superconformal field theories and supergravity," Advances in Theoretical and Mathematical Physics, vol. 2, no. 2, pp. 231-252, 1998.

[2] S. S. Gubser, I. R. Klebanov, and A. M. Polyakov, "Gauge theory correlators from non-critical string theory," Physics Letters B, vol. 428, no. 1-2, pp. 105-114, 1998.

[3] E. Witten, "Anti-de Sitter space and holography," Advances in Theoretical and Mathematical Physics, vol. 2, pp. 253-291, 1998.

[4] J. Erlich, E. Katz, D. T. Son, and M. A. Stephanov, "QCD and a holographic model of hadrons," Physical Review Letters, vol. 95, Article ID 261602, 2005.
[5] A. Karch, E. Katz, D. T. Son, and M. A. Stephanov, "Linear confinement and AdS/QCD," Physical Review D, vol. 74, Article ID 015005, 2006.

[6] T. Sakai and S. Sugimoto, "Low energy hadron physics in holographic QCD," Progress of Theoretical Physics, vol. 113, pp. 843$882,2005$.

[7] T. Sakai and S. Sugimoto, "More on a holographic dual of QCD," Progress of Theoretical Physics, vol. 114, pp. 1083-1118, 2005.

[8] G. Policastro, D. T. Son, and A. O. Starinets, "From AdS/CFT correspondence to hydrodynamics," Journal of High Energy Physics, no. 9, 2002.

[9] G. Policastro, D. T. Son, and A. O. Starinets, "From AdS/CFT correspondence to hydrodynamics. II. Sound waves," Journal of High Energy Physics, no. 12, 2002.

[10] P. Kovtun, D. T. Son, and A. O. Starinets, "Holography and hydrodynamics: diffusion on stretched horizons," Journal of High Energy Physics, no. 10, 2003.

[11] N. Iqbal and H. Liu, "Universality of the hydrodynamic limit in AdS/CFT and the membrane paradigm," Physical Review D, vol. 79, Article ID 025023, 2009.

[12] S. S. Gubser, I. R. Klebanov, and A. W. Peet, "Entropy and temperature of black 3-branes," Physical Review D, vol. 54, no. 6, pp. 3915-3919, 1996.

[13] J. I. Kapusta and T. Springer, "Shear transport coefficients from gauge/gravity correspondence," Physical Review D, vol. 78, Article ID 066017, 2008.

[14] T. Springer, "Sound mode hydrodynamics from bulk scalar fields," Physical Review D, vol. 79, Article ID 046003, 2009.

[15] S. A. Hartnoll, "Lectures on holographic methods for condensed matter physics," Classical and Quantum Gravity, vol. 26, no. 22, Article ID 224002, 2009.

[16] C. P. Herzog, "Lectures on holographic superfluidity and superconductivity," Journal of Physics A, vol. 42, no. 34, Article ID 343001, 2009.

[17] J. McGreevy, "Holographic duality with a view toward manybody physics," Advances in High Energy Physics, vol. 2010, Article ID 723105, 54 pages, 2010.

[18] G. T. Horowitz, "Theory of superconductivity," Lecture Notes in Physics, vol. 828, pp. 313-347, 2011.

[19] S. Sachdev, "Condensed matter and AdS/CFT," Lecture Notes in Physics, vol. 828, pp. 273-311, 2011.

[20] S. S. Gubser, "Breaking an Abelian gauge symmetry near a black hole horizon," Physical Review D, vol. 78, Article ID 065034, 2008 .

[21] S. A. Hartnoll, C. P. Herzog, and G. T. Horowitz, "Building a holographic superconductor," Physical Review Letters, vol. 101, no. 3, Article ID 031601, 2008.

[22] S. S. Gubser and S. S. Pufu, "The gravity dual of a $R$-wave superconductor," Journal of High Energy Physics, no. 11, 2008.

[23] S. A. Hartnoll, C. P. Herzog, and G. T. Horowitz, "Holographic superconductors," Journal of High Energy Physics, no. 12, 2008.

[24] J.-W. Chen, Y.-J. Kao, D. Maity, W.-Y. Wen, and C.-P. Yeh, "Towards a holographic model of D-wave superconductors," Physical Review D, vol. 81, Article ID 106008, 2010.

[25] C. P. Herzog, "Analytic holographic superconductor," Physical Review D, vol. 81, no. 12, Article ID 126009, 2010.

[26] M. Ammon, J. Erdmenger, M. Kaminski, and P. Kerner, "Superconductivity from gauge/gravity duality with flavor," Physics Letters B, vol. 680, pp. 516-520. 
[27] M. Ammon, J. Erdmenger, P. Kerner, and M. Kaminski, "Flavor superconductivity from gauge/gravity duality," Journal of High Energy Physics, no. 10, 2009.

[28] S. S. Gubser, C. P. Herzog, S. S. Pufu, and T. Tesileanu, "Superconductors from superstrings," Physical Review Letters, vol. 103, no. 14, Article ID 141601, 2009.

[29] J. P. Gauntlett, J. Sonner, and T. Wiseman, "Holographic superconductivity in M theory," Physical Review Letters, vol. 103, no. 15, Article ID 151601, 2009.

[30] S. A. Hartnoll, J. Polchinski, E. Silverstein, and D. Tong, "Towards strange metallic holography," Journal of High Energy Physics, vol. 2010, no. 4, article 120, 2010.

[31] B.-H. Lee, S. Nam, D.-W. Pang, and C. Park, "Conductivity in an anisotropic background," Physical Review D, vol. 83, Article ID 066005, 2011.

[32] B.-H. Lee, D.-W. Pang, and C. Park, "Strange metallic behavior in anisotropic background," Journal of High Energy Physics, vol. 2010, article 57, 2010.

[33] S. Kulkarni, B.-H. Lee, C. Park, and R. Roychowdhury, "Nonconformal hydrodynamics in Einstein-dilaton theory," Journal of High Energy Physics, vol. 2012, article 4, 2012.

[34] J. Bhattacharya, M. Nozaki, T. Takayanagi, and T. IJgajin, "On holography with hyperscaling violation," Physical Review Letters, vol. 110, no. 9, Article ID 091602, 2013.

[35] S. S. Gubser, "Curvature singularities: the good, the bad, and the naked," Advances in Theoretical and Mathematical Physics, vol. 4, no. 3, pp. 679-745, 2000.

[36] B. Gouteraux and E. Kiritsis, "Generalized holographic quantum criticality at finite density," Journal of High Energy Physics, vol. 2011, article 36, 2011.

[37] K. Goldstein, S. Kachru, S. Prakash, and S. P. Trivedi, "Holography of charged dilaton black holes," Journal of High Energy Physics, vol. 2010, article 78, 2010.

[38] C. Charmousis, B. Gouteraux, B. S. Kim, E. Kiritsis, and R. Meyer, "Effective holographic theories for low-temperature condensed matter systems," Journal of High Energy Physics, vol. 2010, article 151, 2010.

[39] M. Cadoni, S. Mignemi, and M. Serra, "Exact solutions with AdS asymptotics of Einstein and Einstein-Maxwell gravity minimally coupled to a scalar field," Physical Review D, vol. 84, Article ID 084046, 2011.

[40] K. Goldstein, N. Iizuka, S. Kachru, S. Prakash, S. P. Trivedi, and A. Westphal, "Holography of dyonic dilaton black branes," Journal of High Energy Physics, vol. 2010, no. 10, article 027, 2010.

[41] X. Dong, S. Harrison, S. Kachru, G. Torroba, and H. Wang, "Aspects of holography for theories with hyperscaling violation," Journal of High Energy Physics, vol. 2012, article 41, 2012.

[42] L. Huijse, S. Sachdev, and B. Swingle, "Hidden Fermi surfaces in compressible states of gauge-gravity duality," Physical Review $B$, vol. 85, Article ID 035121, 14 pages, 2012.

[43] B. S. Kim, "Schrödinger holography with and without hyperscaling violation," Journal of High Energy Physics, vol. 1206, p. 116, 2012.

[44] E. Perlmutter, "Hyperscaling violation from supergravity," Journal of High Energy Physics, vol. 1206, p. 165, 2012.

[45] H. J. Boonstra, K. Skenderis, and P. K. Townsend, "The domainwall/QFT correspondence," Journal of High Energy Physics, vol. 3, no. 1, 1999 .

[46] I. Kanitscheider, K. Skenderis, and M. Taylor, "Precision holography for non-conformal branes," Journal of High Energy Physics, no. 9, 2008.
[47] I. Kanitscheider and K. Skenderis, "Universal hydrodynamics of non-conformal branes," Journal of High Energy Physics, no. 4, 2009.

[48] B. Goutéraux, J. Smolic, M. Smolic, K. Skenderis, and M. Taylor, "Holography for Einstein-Maxwell-dilaton theories from generalized dimensional reduction," Journal of High Energy Physics, vol. 2012, article 89, 2012.

[49] V. Balasubramanian and P. Kraus, "A stress tensor for anti-de Sitter gravity," Communications in Mathematical Physics, vol. 208, no. 2, pp. 413-428, 1999.

[50] A. Batrachenko, J. T. Liu, R. McNees, W. A. Sabra, and W. Y. Wen, "Black hole mass and Hamilton-Jacobi counterterms," Journal of High Energy Physics, no. 5, 2005.

[51] D. T. Son and A. O. Starinets, "Hydrodynamics of R-charged black holes," Journal of High Energy Physics, no. 3, 2006.

[52] S. Kulkarni, B.-H. Lee, J.-H. Oh, C. Park, and R. Roychowdhury, "Transports in non-conformal holographic fluids," Journal of High Energy Physics, vol. 1303, p. 149, 2013.

[53] S. S. Gubser, "Dilaton-driven confinement," http://arxiv.org/ abs/hep-th/9902155.

[54] D. Bak, M. Gutperle, S. Hirano, and N. Ohta, "Dilatonic repulsons and confinement via the AdS/CFT correspondence," Physical Review D, vol. 70, no. 8, Article ID 086004, 2004.

[55] S. S. Gubser, "Drag force in AdS/CFT"' Physical Review D, vol. 74, no. 12, Article ID 126005, 2006.

[56] E. Kiritsis, "Lorentz violation, gravity, dissipation and holography," Journal of High Energy Physics, vol. 2013, article 30, 2013.

[57] M. Alishahiha and H. Yavartanoo, "On holography with hyperscaling violation," Journal of High Energy Physics, vol. 2012, article 34, 2012.

[58] S. Ryu and T. Takayanagi, "Holographic derivation of entanglement entropy from the anti-de Sitter space/conformal field theory correspondence," Physical Review Letters, vol. 96, no. 18, Article ID 181602, 2006.

[59] S. Ryu and T. Takayanagi, "Aspects of holographic entanglement entropy," Journal of High Energy Physics, vol. 2006, no. 8, article 45, 2006. 

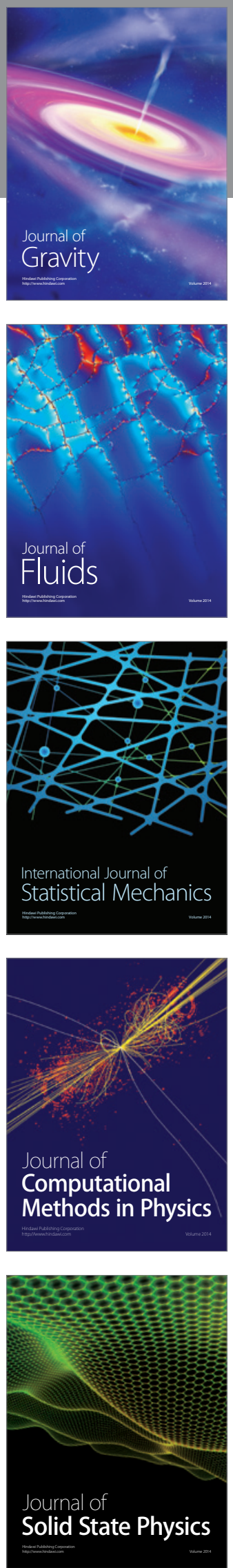

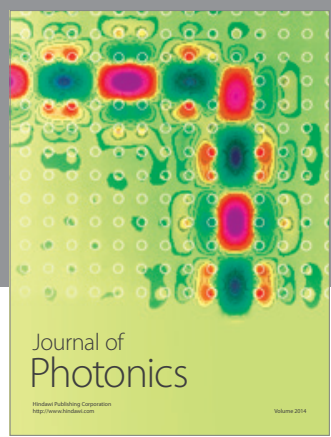

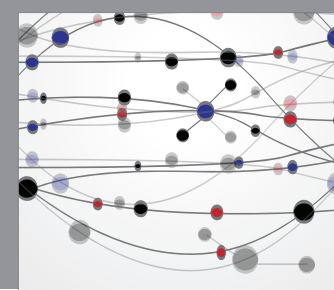

The Scientific World Journal

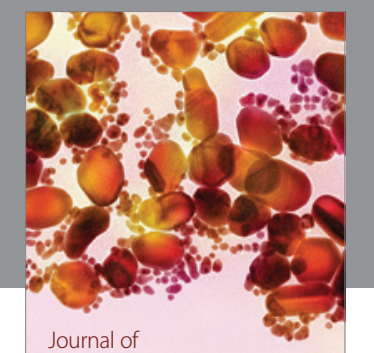

Soft Matter
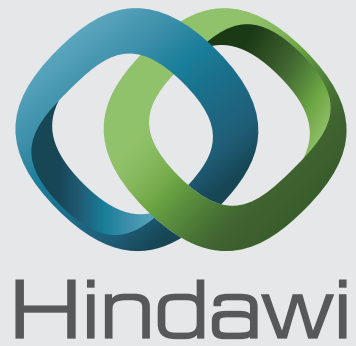

Submit your manuscripts at

http://www.hindawi.com
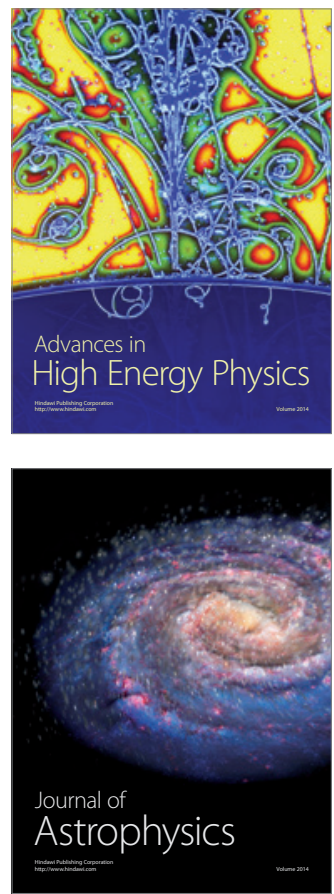
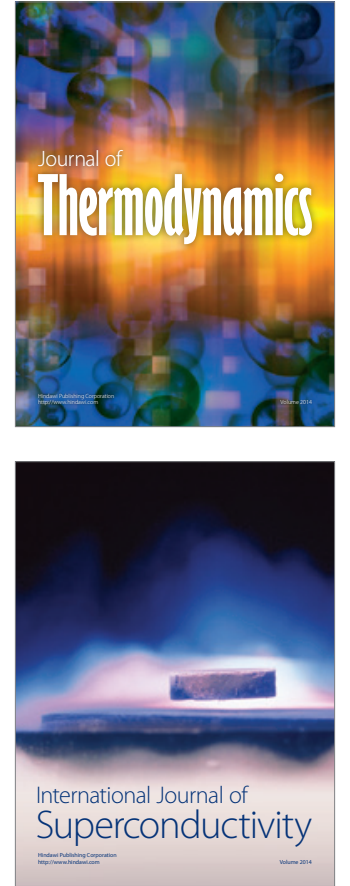
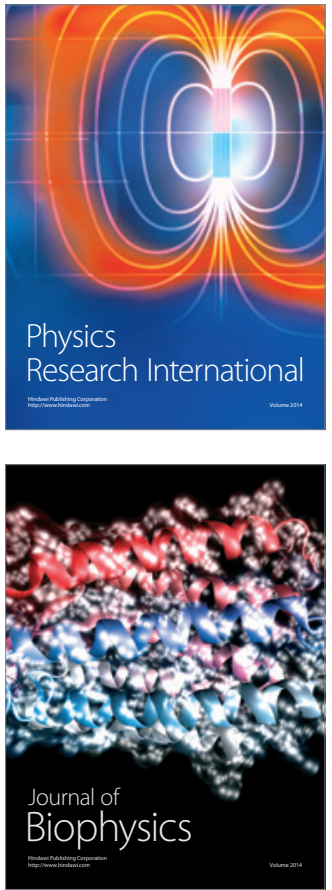
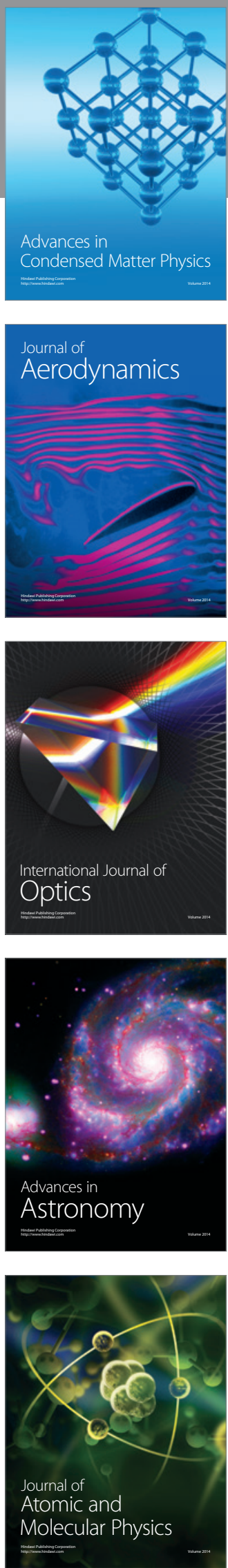